\title{
Tandem CAR T cells targeting HER2 and IL13R $\alpha 2$ mitigate tumor antigen escape
}

\author{
Meenakshi Hegde, ${ }^{1,2,3,4,5}$ Malini Mukherjee, ${ }^{3,4,6}$ Zakaria Grada, ${ }^{1,2,3,4}$ Antonella Pignata, ${ }^{1,2,3,4}$ Daniel Landi, ${ }^{1,2,3,4}$ Shoba A. Navai, ${ }^{1,2,3,4}$ \\ Amanda Wakefield, ${ }^{1,2,3,4}$ Kristen Fousek, ${ }^{1,2,3,4,5}$ Kevin Bielamowicz, ${ }^{1,2,3,4}$ Kevin K.H. Chow, ${ }^{1,2,3,4}$ Vita S. Brawley, ${ }^{1,2,3,4}$ \\ Tiara T. Byrd, ${ }^{1,2,3,4,5}$ Simone Krebs, ${ }^{1,2,3,4}$ Stephen Gottschalk, ${ }^{1,2,3,4,5}$ Winfried S. Wels, ${ }^{7}$ Matthew L. Baker, ${ }^{8}$ Gianpietro Dotti, ${ }^{1,2,3}$ \\ Maksim Mamonkin, ${ }^{1,2,3,4}$ Malcolm K. Brenner, ${ }^{1,2,3,4}$ Jordan S. Orange, ${ }^{3,4,6}$ and Nabil Ahmed ${ }^{1,2,3,4,5}$ \\ 'Center for Cell and Gene Therapy, Texas Children's Hospital, Houston Methodist Hospital, Baylor College of Medicine, Houston, Texas, USA. ${ }^{2}$ Texas Children's Cancer and Hematology Centers and \\ ${ }^{3}$ Texas Children's Hospital, Baylor College of Medicine, Houston, Texas, USA. ${ }^{4}$ Department of Pediatrics and 5 Interdepartmental Program in Translational Biology and Molecular Medicine, \\ Baylor College of Medicine, Houston, Texas, USA. ${ }^{6}$ Texas Children's Hospital Center for Human Immunobiology, Houston, Texas, USA. 'Ceorg-Speyer-Haus, Institute for Tumor Biology and \\ Experimental Therapy, Frankfurt, Germany. ${ }^{8}$ National Center for Macromolecular Imaging, Department of Biochemistry, Baylor College of Medicine, Houston, Texas, USA.
}

\begin{abstract}
In preclinical models of glioblastoma, antigen escape variants can lead to tumor recurrence after treatment with CAR T cells that are redirected to single tumor antigens. Given the heterogeneous expression of antigens on glioblastomas, we hypothesized that a bispecific CAR molecule would mitigate antigen escape and improve the antitumor activity of T cells. Here, we created a CAR that joins a HER2-binding SCFv and an IL13Ro2-binding IL-13 mutein to make a tandem CAR exodomain (TanCAR) and a CD28. endodomain. We determined that patient TanCAR T cells showed distinct binding to HER2 or IL13R $\alpha 2$ and had the capability to lyse autologous glioblastoma. TanCAR T cells exhibited activation dynamics that were comparable to those of single CAR T cells upon encounter of HER2 or IL13R 2 2. We observed that TanCARs engaged HER2 and IL13R $\alpha 2$ simultaneously by inducing HER2-IL13R $\alpha 2$ heterodimers, which promoted superadditive T cell activation when both antigens were encountered concurrently. TanCAR T cell activity was more sustained but not more exhaustible than that of T cells that coexpressed a HER2 CAR and an IL13R 22 CAR, T cells with a unispecific CAR, or a pooled product. In a murine glioblastoma model, TanCAR T cells mitigated antigen escape, displayed enhanced antitumor efficacy, and improved animal survival. Thus, TanCAR T cells show therapeutic potential to improve glioblastoma control by coengaging HER2 and IL13R 2 in an augmented, bivalent immune synapse that enhances $\mathrm{T}$ cell functionality and reduces antigen escape.
\end{abstract}

\section{Introduction}

Adoptive transfer of chimeric antigen receptor-grafted (CARgrafted) (1) T cells has induced tumor regression in several preclinical models of glioblastoma (GBM) $(2-4)$, osteosarcoma $(5,6)$, and neuroblastoma (7). However, only sporadic clinical responses have been observed in early-phase clinical trials for these tumors (8-11). In contrast, the sustained remission seen in preclinical models of CAR T cell transfer in B cell leukemia was successfully translated to favorable outcomes in early clinical trials. These successes were achieved by targeting of CD19, a B-cell lineage marker that is uniformly expressed in B cell precursor acute lymphoblastic leukemia and chronic lymphocytic leukemia cells (12-19). Explanations for this discrepancy include but are not limited to transient $\mathrm{T}$ cell persistence in vivo, modest $\mathrm{T}$ cell homing, and inadequate $\mathrm{T}$ cell activation and/or $\mathrm{T}$ cell inhibition at the tumor site $(8,9)$. The limited spectrum of $\mathrm{T}$ cell specificity in the face of the heterogeneous and potentially dynamic antigen landscape is perhaps the biggest challenge for CAR T cell therapy for solid tumors (20-24).

Conflict of interest: M. Hegde, S. Gottschalk, Z. Grada, V.S. Brawley, T.T. Byrd, and N. Ahmed have patent applications in the field of T cell and gene-modified T cell therapy for cancer.

Submitted: June 22, 2015; Accepted: May 13, 2016.

Reference information: J Clin Invest. 2016;126(8):3036-3052. doi:10.1172/JCI83416.
We previously reported on GBM's markedly heterogeneous antigenic landscape (20). A mathematical model of the expression hierarchy of 3 validated glioma antigens (21, 25-28), HER2, IL13R $\alpha 2$, and EphA2, predicted enhanced odds of tumor elimination on targeting of any 2 of these 3 antigens (20). Specifically, while targeting HER2 or IL13R $\alpha 2$ alone predicted a $60 \%-70 \%$ probability of near-complete tumor elimination, simultaneously targeting HER2 and IL13R $\alpha 2$ was predicted to eliminate more than $90 \%$ in a cohort of 20 primary GBMs (20).

We reasoned that a single CAR molecule with docking capacity to 2 tumor-associated antigens (TAAs) will form a bivalent $\mathrm{T}$ cell/GBM immunological synapse (IS), enhancing $\mathrm{T}$ cell activation and offsetting antigen escape, and collectively, these attributes will translate into superior antitumor activity (29).

We report on a bispecific CAR molecule that incorporates 2 antigen recognition domains for HER2 and IL13R $\alpha 2$, joined in tandem, thus termed TanCAR (29). We describe the design, in silico modeling, and super-resolution imaging of the TanCAR IS with GBM cells, and show functional superiority of T cells expressing TanCARs ex vivo and in an orthotopic GBM xenograft model.

\section{Results}

Antigen escape variants prevail in GBM recurrences after CAR T cell therapy. GBM exhibits substantial genetic as well as antigenic het- 
A

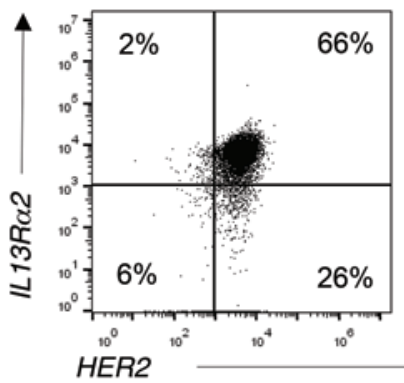

UPN 2

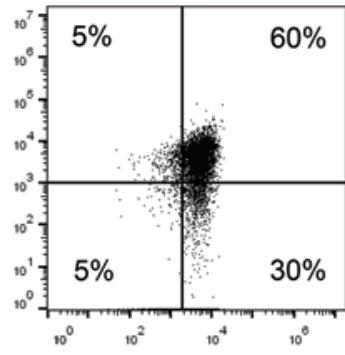

UPN 3

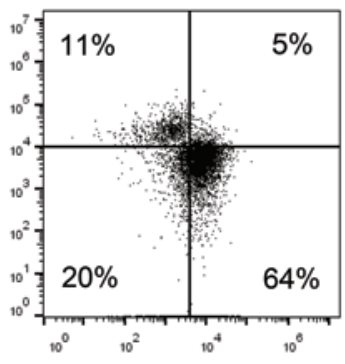

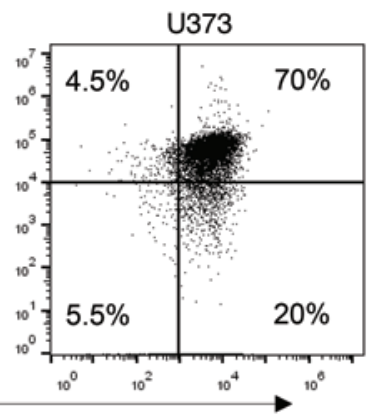

IL13R $\alpha 2$
B
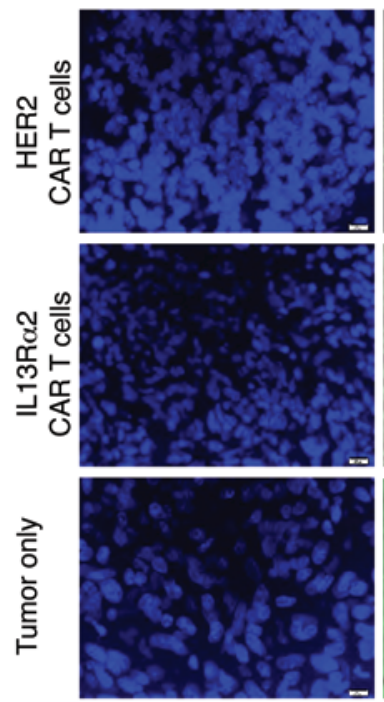

HER2
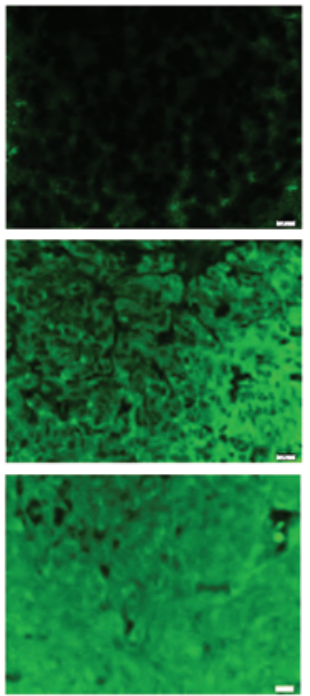
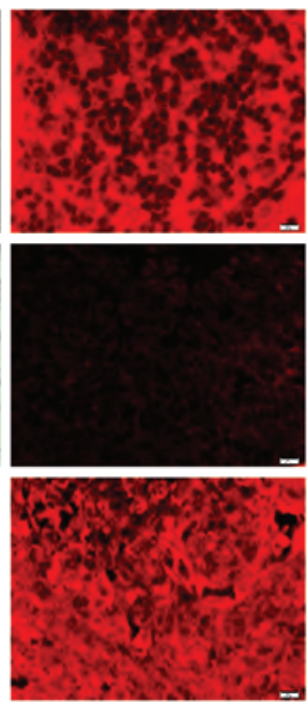

C

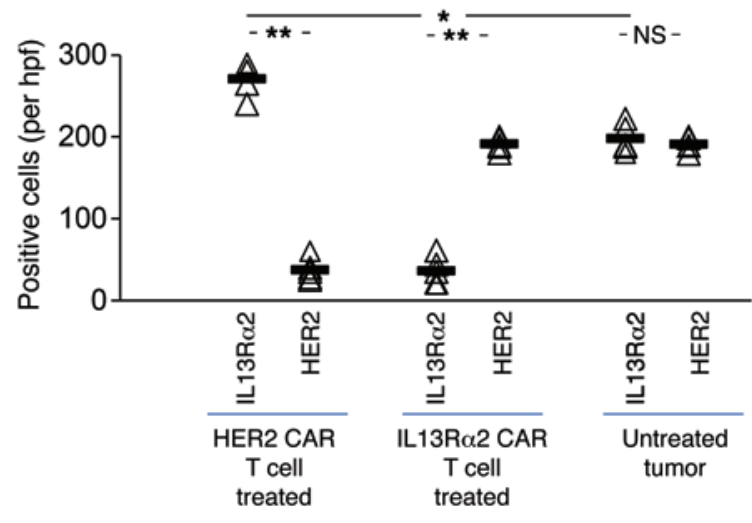

Figure 1. Surface expression of HER2 and IL13R $\alpha 2$ in primary GBM and the GBM cell line U373 and loss of target antigen in CAR T cell-treated xenografts. (A) Single-cell suspensions of primary GBM excision samples and U373 were costained for HER2 and IL13R $\alpha 2$, and more than 100,000 events were analyzed by flow cytometry. Shown are representative dot plots of 3 experiments. UPN, unique patient number. (B) Analysis of U373 xenografts recurring after CAR T cell therapy targeting HER2 and IL13R $\alpha 2$ using coimmunofluorescence for HER2 and IL13R $\alpha 2$. Original magnification, $\times 100$. Scale bar $-20 \mu \mathrm{m}$. (C) Quantification of staining for HER2 and IL13R $\alpha 2$ of the data shown in B. Cells were counted in 5 high-power fields (hpfs) per sample. Individual values per hpf and average (bar) are shown. A single-step Tukey's range test was used for multiple comparisons. ${ }^{*} P<0.05,{ }^{* *} P<0.005$.

erogeneity. We and others have shown that experimental orthotopic GBM regresses after administration of HER2 or IL13R $\alpha 2$ CAR $\mathrm{T}$ cells, yet tumors recur in $40 \%-60 \%$ of CAR T cell-treated animals $(2-4,30)$. Therefore, we assessed the surface expression of HER2 and IL13R $\alpha 2$ in a cohort of 3 primary GBM samples (unique patient numbers 1-3 [UPN 1-UPN 3]) obtained from surgical excision material (hereafter referred to as primary GBM). Consistent with our previous results, variable HER2 and IL13R $\alpha 2$ expression was observed (Figure 1A). While UPN 1 and 2 had a predominantly HER2- and IL13R $\alpha 2$-coexpressing tumor cell population (66\% and $60 \%$, respectively), UPN 3 had 2 distinct tumor cell populations with a predominant positivity for HER2 (64\%). IL13R $\alpha 2$ expression was only $11 \%$, with $5 \%$ of the cells coexpressing both antigens.

We studied the expression of glioma antigens in recurrent U373 (a HER2+, IL13R $\alpha 2+$ human GBM cell line) xenografts after adoptive transfer of either HER2 or IL13R $\alpha 2$ CAR T cells using coimmunofluorescence for both HER2 and IL13R $\alpha 2$ Recurrences after HER2 CAR T cell transfer showed dim to undetectable HER2 expression. Similarly, tumors recurring after IL13R $\alpha 2$ CAR T cell transfer showed dim staining for IL13R $\alpha 2$. Interestingly, residual and recurrent tumors maintained and even intensified IL13R $\alpha 2$ expression after treatment with HER2 CAR T cells. Conversely, tumors maintained HER2 expression after IL13R $\alpha 2$ targeting (Figure 1, B and C). Escape variants prevailed in all recurrences while a second targetable antigen was preserved.

Construction and in silico interrogation of the structural functionality of a HER2/IL13R $\alpha 2$ bivalent CAR molecule. In order to redirect the specificity of T cells toward both HER 2 and IL13R $\alpha 2$ simultaneously using a single CAR molecule, we constructed a TanCAR: a bivalent CAR molecule that can engage both TAAs by a single exodomain. The CAR exodomain consists of a 19-amino acid leader/secretory sequence (L), followed by a high-affinity IL-13 mutein that is separated from the HER2-specific FRP5-scFv by a 15-amino acid glycine/ serine repeat linker (Figure 2A and ref. 29). The composite cell binding domain is followed by an IgG1 hinge moiety (H). A CD28 transmembrane domain tethers this exodomain to a second-generation endodomain incorporating the CD28 and CD3 $\zeta$ signaling chains. The encoding transgene was codon-optimized, then synthesized and packaged into a $P S F G$ retroviral backbone (Figure $2 \mathrm{~B}$ ).

A model for both the individual domains and the entire TanCAR was constructed using the Phyre2 and RaptorX structure prediction websites (Figure $2 \mathrm{C}$ ). The model suggested that each 
A

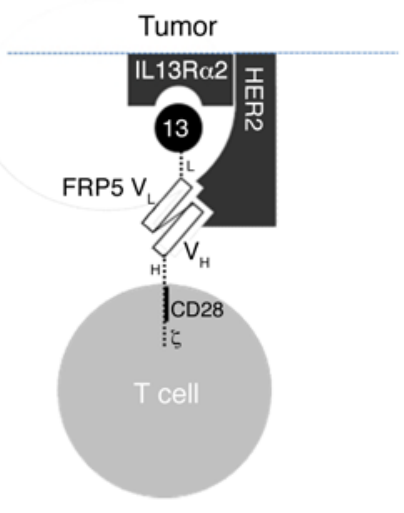

C

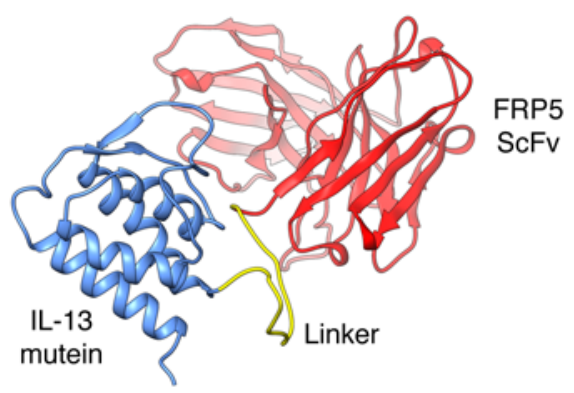

E

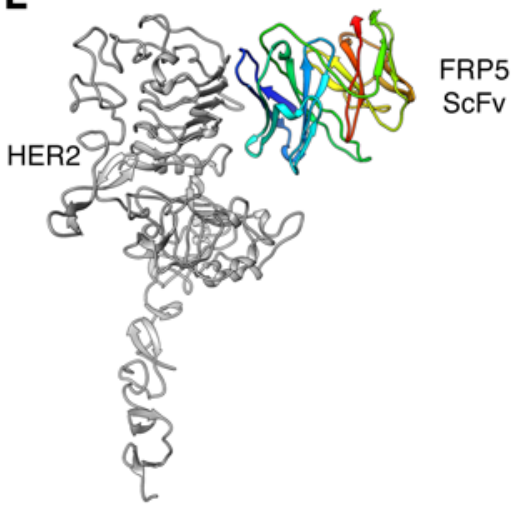

B

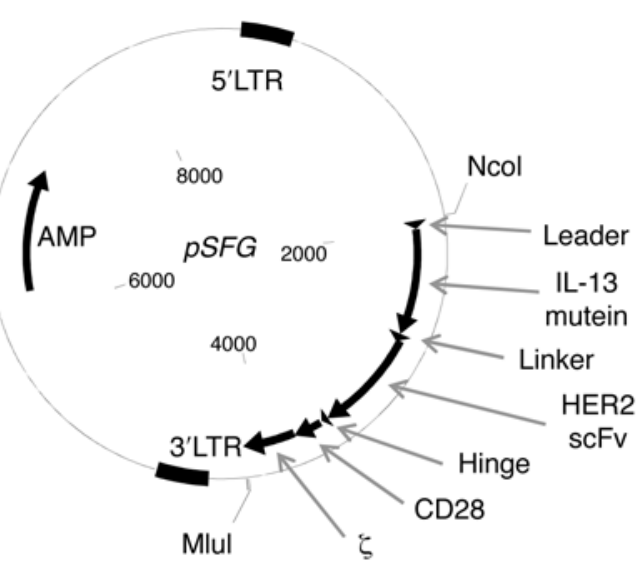

D
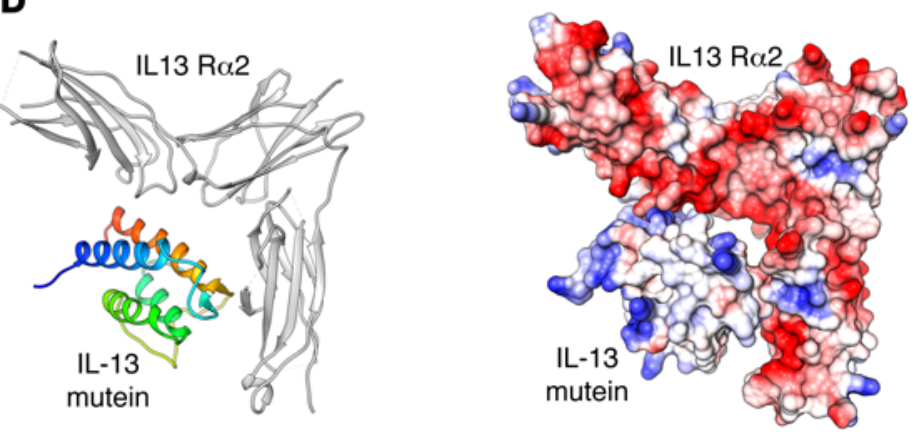

$\mathbf{F}$

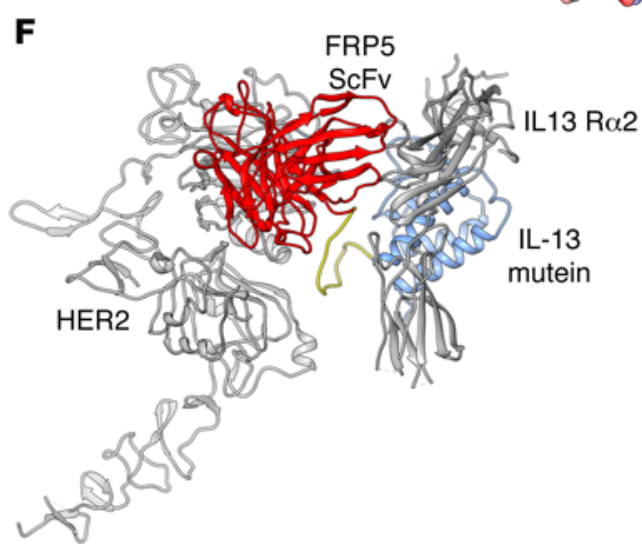

Figure 2. The HER2/IL13R $\alpha 2$ tandem CAR (TanCAR) structure, its encoding transgene, and in silico interrogation of its docking properties. (A) Cartoon depicting the TanCAR docking to respective targets. 13, IL-13 mutein; L, linker; $V_{L}$, FRP5 variable domain of the Ig light chain; $V_{H}$, FRP5 variable domain of the Ig heavy chain; H, hinge. (B) SFG vector encoding the HER2/IL13R $\alpha 2$ TanCAR. (C-F) In silico modeling of HIL TanCAR to respective targets: (C) A computational rendition of the TanCAR structure. The FRP5-scFv is shown in red, while the IL-13 mutein domain is shown in blue. The glycine/serine linker is highlighted in yellow. (D) The computational docking of the IL-13 mutein domain to IL13R $\alpha 2$. The receptor is colored in gray, while the IL-13 mutein domain is rainbow colored (blue to red, $\mathrm{N}$ - to C-terminus). The IL-13 receptor is shown with its C-terminus pointing down. The docking model shows good electrostatic complementarity, where red is negatively charged and blue positively charged, between the 2 molecules. (E) Docking of the FRP5-scFv to the HER2 receptor. Coloring is as in D; the scFv domain is rainbow colored, and the receptor is gray. (F) A favorable model of the TanCAR bound to both HER2 and IL13R $\alpha 2$.

domain was structurally discrete; the IL-13 mutein domain contained a 4-helical cytokine fold, while the FRP5-scFv domain had an Ig-like $\beta$-sandwich fold $(2,3)$. The model for the entire TanCAR did not suggest any interaction between the IL-13 mutein and the FRP5-scFv domain, as the intervening glycine/serine linker appears to separate the 2 domains. Further, this model suggested a linear arrangement of the domains, in which the FRP5scFv domain is anchored closer to the membrane than the IL-13 mutein. Importantly, the model suggested that the receptor binding moieties for each of the domains are likely available to the cog- nate receptors, though distal arrangement of the IL-13 mutein may allow for a more exposed IL13R $\alpha 2$ epitope. As there are minimal interactions between the domains and a relatively long glycine/ serine linker separates them, it was unclear whether this architecture would persist in vivo.

Docking of the TanCAR to the cognate receptors was initially modeled for the individual IL-13 mutein and the FRP5-scFv (Figure 2, D and E, respectively). Models for IL-13/IL13R $\alpha 2$ (Protein Data Bank ID: 3LB6) and HER2/FRP5-scFv were individually optimized with Rosetta Dock, then combined in the context of the 


\begin{tabular}{|c|c|c|}
\hline Score & IL-13 mutein docking & FRP5 docking \\
\hline Total score & -268.21 (6th) & -462.853 (8th) \\
\hline RMS & 4.153 & 10.598 \\
\hline Fnat & 0.491 & 0.103 \\
\hline Isc & -7.011 (3rd) & -4.131 (14th) \\
\hline Irms & 2.431 & 4.191 \\
\hline cen_rms & 4.314 & 7.951 \\
\hline dslf_ca_dih & 4.061 & 4.336 \\
\hline dslf_cs_ang & 24.729 & 67.78 \\
\hline dslf_ss_dih & 37.862 & 56.87 \\
\hline dslf_ss_dst & -12.38 & -35.553 \\
\hline fa_atr & -581.05 & $-1,238.48$ \\
\hline fa_dun & 30.17 & 57.804 \\
\hline fa_elec & -3.563 & -8.558 \\
\hline fa_pair & -5.908 & -17.211 \\
\hline fa_rep & 35.166 & 196.782 \\
\hline fa_sol & 232.575 & 528.028 \\
\hline hbond_bb_sc & -4.472 & -13.712 \\
\hline hbond_Ir_bb & -14.077 & -38.51 \\
\hline hbond_sc & -3.123 & -7.173 \\
\hline hbond_sr_bb & -8.2 & -15.256 \\
\hline interchain_contact & -7 & -20 \\
\hline interchain_env & -34.358 & -56.314 \\
\hline interchain_pair & 1.385 & -1.162 \\
\hline interchain_vdw & 0.115 & 0.001 \\
\hline st_rmsd & 4.728 & 7.277 \\
\hline
\end{tabular}

full TanCAR (Figure 2F) (29). The arrangement of the TanCAR domains allowed for binding both receptors, simultaneously. It is likely that the potentially flexible linker between the domains would permit conformational variability of domain arrangement, thus allowing the IL-13 mutein and the FRP5-scFv to optimize the simultaneous binding of the 2 cognate receptors. Our computational modeling is indicative of 1 such arrangement. Table 1 shows the energy analysis of TanCAR docking.

TanCAR distinctly binds HER2 and IL13R 2 and mediates enhanced $T$ cell reactivity against autologous GBM and GBM cell lines. A retroviral system was used to express TanCAR onto primary human T cells from GBM patients (2). We used soluble HER2 and IL13R $\alpha 2$ to confirm the distinct unispecific as well as bispecific binding of the TanCAR exodomain to both TAAs (Supplemental Figure 1A; supplemental material available online with this article; doi:10.1172/ JCI83416DS1). The high concordance between the juxtamembranous FRP5-scFv and the IL-13 mutein conjugates confirmed that the TanCAR molecule exodomain surfaced on the T cells in its entirety. Expression was stable for more than 12 weeks in ex vivo cultures.

We performed a coculture assay to test whether encountering autologous GBM cells would induce T cell activation. TanCAR T cells from UPN 1, 2, and 3 specifically secreted IFN- $\gamma$ and IL-2 upon encountering autologous GBM cells. The levels of IFN- $\gamma$ and IL-2 were undetectable (significantly lower in UPN 2) when nontransduced (NT) T cells were used as effectors (Figure 3A). The background reactivity seen with UPN 2 NT T cells is likely due to a less than $5 \%$ subpopulation of NK cells detected in the product.

The ability to engage both HER2 and IL13R $\alpha 2$ could provide an advantage to $\mathrm{T}$ cell effectors over their unispecific counterparts by increasing the overall avidity of the interaction. We assessed the cytolytic ability of TanCAR T cells, HER2 CAR T cells, IL13R $\alpha 2$ CAR T cells, and equal aliquots of both unispecific CAR T cells pooled together (CARpool) and bi-expressers of both HER2 and IL13R $\alpha 2$ CAR (biCAR) generated from patients UPN 1, 2 , and 3 (Figure $3 \mathrm{~B}$ ). In all subsequent experiments, the TanCAR transduction rate was normalized to the HER2 CAR- and IL13R $\alpha 2$ CAR-coexpressing $\mathrm{T}$ cell population in the biCAR $\mathrm{T}$ cell product and to individual CAR-expressing $\mathrm{T}$ cell population in unispecific T cell products (Supplemental Figure 1B). Significantly higher killing at most effector-to-target ratios was seen with UPN 1, 2, and 3 TanCAR T cells compared with unispecific or biCAR T cells. UPN 3 IL13R $\alpha 2$ CAR T cells exhibited only modest cytolytic activity, likely because only $16 \%$ of this tumor's cells expressed IL13R $\alpha 2$. Similar results were seen when donor-derived $\mathrm{T}$ cells encountered the GBM cell line U373. There was no cytolytic activity by NT primary $\mathrm{T}$ cell blasts from the same patient and no significant cytolytic activity of TanCAR T cells against HER2- and IL13R $\alpha 2$-null targets (not shown).

In order to assess their relative cytokine secretion capacity, we cocultured U373 glioma cells with TanCAR, biCAR, HER2, IL13ra2, and CARpool T cells at a ratio of $1 \mathrm{~T}$ cell to 4 tumor cells. Supernatants were collected at 24 hours and analyzed for IL-2 and IFN- $\gamma$ using ELISA. We detected significantly higher concentrations of cytokines in supernatants in samples with TanCAR T cells as effectors compared with all other T cell products. Neither NT cells nor U373 alone had detectable levels of cytokines (Figure 3C).

TanCAR $T$ cells are activated in a dose-dependent manner upon contact with either HER2 or IL13R 22 and show superadditive activation upon encountering both HER 2 and IL13R $\alpha 2$. To test the specificity of TanCAR T cells and their activation dynamics, we cultured them on plates coated with increasing densities of HER2.Fc, IL13R $\alpha 2 . F c$, an irrelevant target (GD2.scFv anti-idiotype), and a nonspecific $\mathrm{T}$ cell receptor stimulant (OKT3). We compared their activation dynamics to those of biCAR and HER2- and IL13R $\alpha 2-$ unispecific CAR T cells. The supernatant was analyzed at 24 hours for IFN- $\gamma$ and IL-2. TanCAR T cells released IFN- $\gamma$ upon encountering HER2 or IL13R $\alpha 2$ (Figure 4). The magnitude of response was dose-dependent $\left(r^{2} 0.85-0.98\right)$ for both target antigens at low to intermediate density of immobilized HER2.Fc or IL13R $\alpha 2$.Fc. At high antigen densities, a decline in cytokine release was noted. In contrast, cytokine production was significantly higher when TanCAR $\mathrm{T}$ cells encountered both TAAs simultaneously than either antigen alone at any antigen density $(P<0.001)$, and higher than the sum of maximal cytokine concentrations upon single antigen contact, denoting a superadditive effect of encountering both antigen molecules. In contrast, biCAR T cells exhibited similar activation dynamics upon encounter of individual antigens and a simple additive enhanced activation upon encounter of low to intermediate densities of both antigens. At high antigen densities, we saw a decline in both IFN- $\gamma$ and IL-2 production by biCAR T cells. Unispecific CAR T cell products exhibited uniaxial activation dynamics with decline of cytokine production at high antigen 
A
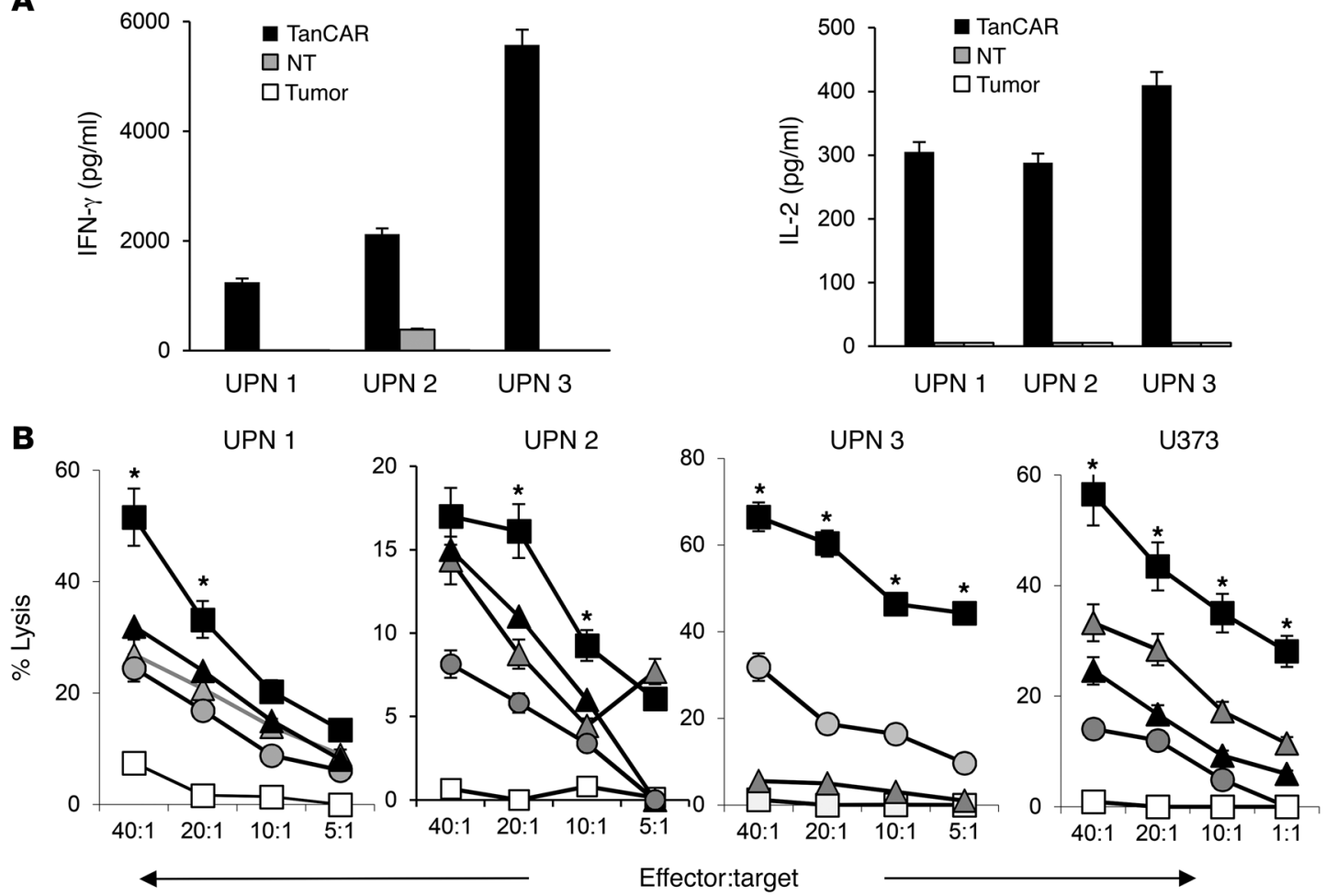

- TanCAR

$\Delta$ biCAR

C
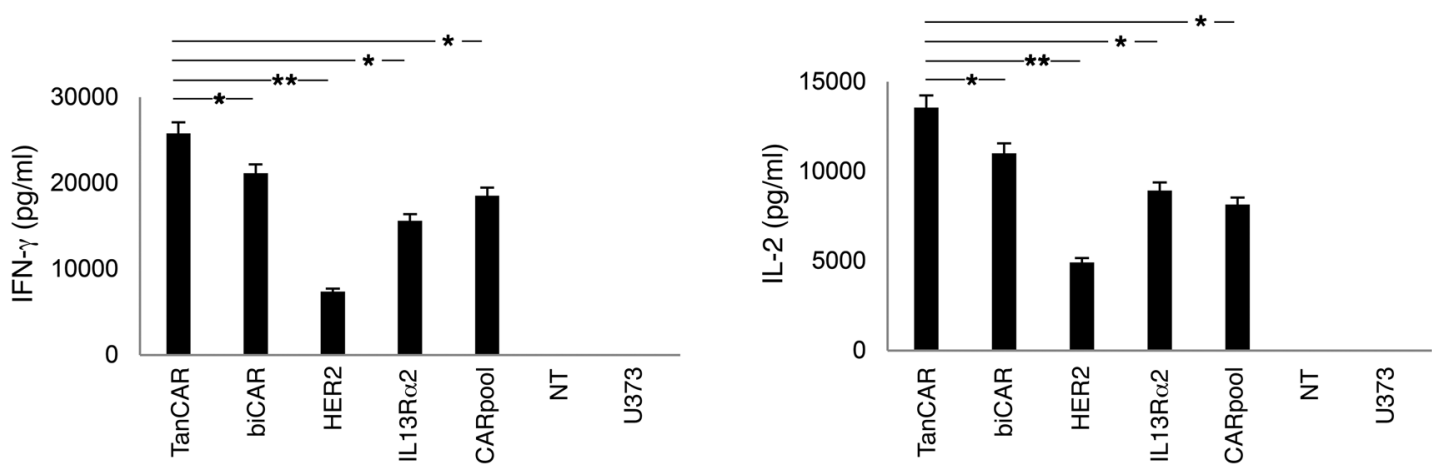

Figure 3. Activity of GBM patients' TanCAR T cells against autologous GBM and U373. (A) Cytokine (IFN- $\gamma$ and IL-2) production of TanCAR and nontransduced (NT) T cells generated from GBM patients detected in the supernatant 24 hours after coculture with autologous primary GBM cells. Shown are pooled data from 2 experiments done in triplicates. (B) Four-hour ${ }^{51} \mathrm{Cr}$-release assays of primary TanCAR T cells from UPN 1, 2, and 3 against autologous GBM cells compared with the unispecific CAR T cells, a pooled product of aliquots thereof (CARpool), T cells coexpressing both HER2 and IL13R 22 CARs (biCAR), and NT T cells, all generated from the same patient. Healthy donor-derived T cells were tested against the U373-CBM line. biCAR T cell product for UPN 3 was not available. Shown are representative data from 3 independent experiments done in triplicates. Two-tailed $t$ test was performed between TanCAR and the T cell product exhibiting the highest degree of killing. ${ }^{*} P<0.05$. (C) Cytokine analysis (IFN- $\gamma$ and IL-2) from supernatants of cocultures of primary TanCAR T cells, biCAR T cells, CARpool T cells, and unispecific CAR T cells with HER2 and IL13 $\alpha 2$ expressing U373 GBM cells (25,000 T cells to 100,000 tumor cells), as detected by ELISA. Shown are pooled data from 3 independent experiments done in triplicates. A single-step Tukey's range test was used. ${ }^{*} P<0.05,{ }^{* *} P<0.005$.

densities. T cells did not produce IFN- $\gamma$ or IL-2 during coculture with the irrelevant GD2.scFv anti-idiotype or on uncoated surfaces. As expected, OKT3 (nonspecific positive control) induced cytokine release through stimulation of the native $\mathrm{T}$ cell receptor.

TanCAR T cells exhibit enhanced and sustained ex vivo antitumor activity that is not associated with a more exhausted phenotype. Excessive stimulation of $\mathrm{T}$ cells, such as that seen with TanCAR $\mathrm{T}$ cells upon simultaneous encounter of both HER2 and IL13R $\alpha 2$, can result in an initially accentuated functionality followed by an exhausted phenotype. To compare the duration of antitumor activity by TanCAR, biCAR, CARpool, and unispecific CAR T cells, we used an electrical impedance-based tumor cell culture system (xCELLigence). U373 cells were seeded and allowed to reach confluence before each $\mathrm{T}$ cell product with matched transduction efficiency was added at a ratio of $1 \mathrm{~T}$ cell to 10 seeded U373 cells. A validated impedance-based cell index formula was used to quantify the density of tumor cells (Figure 5A). The realtime killing of U373 glioma cells was assessed in time-lapse over 150 hours. Initially, both tandem and biCAR T cell products killed U373 significantly more effectively compared with HER2- and IL13R $\alpha 2$ CAR T cells and their pooled product. At and beyond 60 hours, however, TanCAR T cells were significantly better able to 


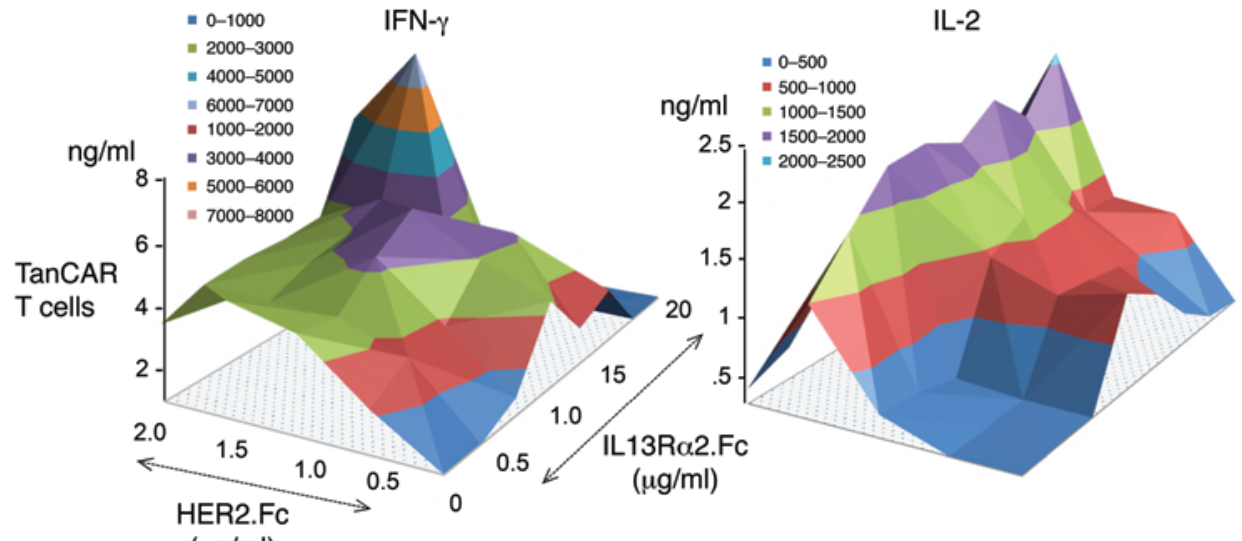

Figure 4. Dynamics of activation of TanCAR T cells upon encounter of a density gradient of HER2 and IL13R $\alpha 2$. The activation dynamics of TanCAR T cells, biCAR T cells, HER2 CAR T cells, and IL13R $\alpha 2$ CAR T cells was studied by seeding of the cells onto polypropylene surface-bound HER2 (range 0-2.0 $\mu \mathrm{g} / \mathrm{ml}$ ), IL13R $\alpha 2$ (range $0-20 \mu \mathrm{g} / \mathrm{ml}$ ), an irrelevant target (CD2.scFv antiidiotype, $5 \mu \mathrm{g} / \mathrm{ml})$, and OKT3 $(1 \mu \mathrm{g} / \mathrm{ml})$. Cytokine analysis (IFN- $\gamma$ and IL-2) of the culture supernatant using ELISA was performed after 24 hours. Shown are representative data from 3 independent experiments done in triplicates. The titration included 10 antigen concentrations on each axis. Two-tailed $t$ test
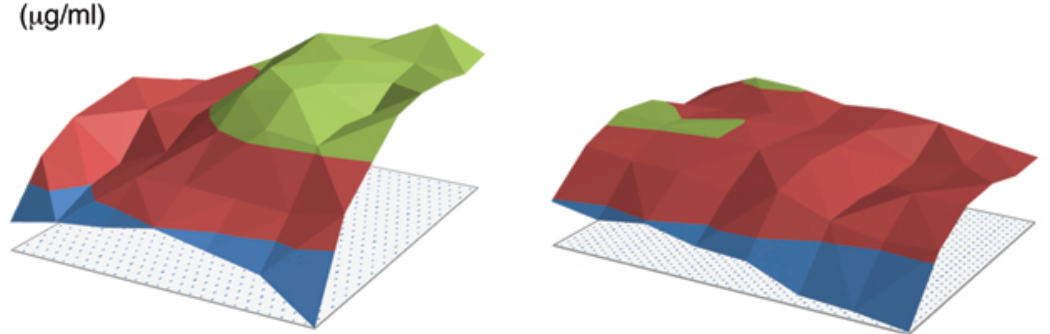
was used to compare TanCAR cytokine values with those for each single-specificity CAR and biCAR T cells. Significant results are reported in Results. Nonspecific positive control OKT3 induced both IFN- $\gamma(5.5 \mathrm{ng} / \mathrm{ml})$ and IL-2 $(2.0 \mathrm{ng} / \mathrm{ml})$ release by TanCAR T cells while there was no cytokine production with GD2 anti-idiotype.
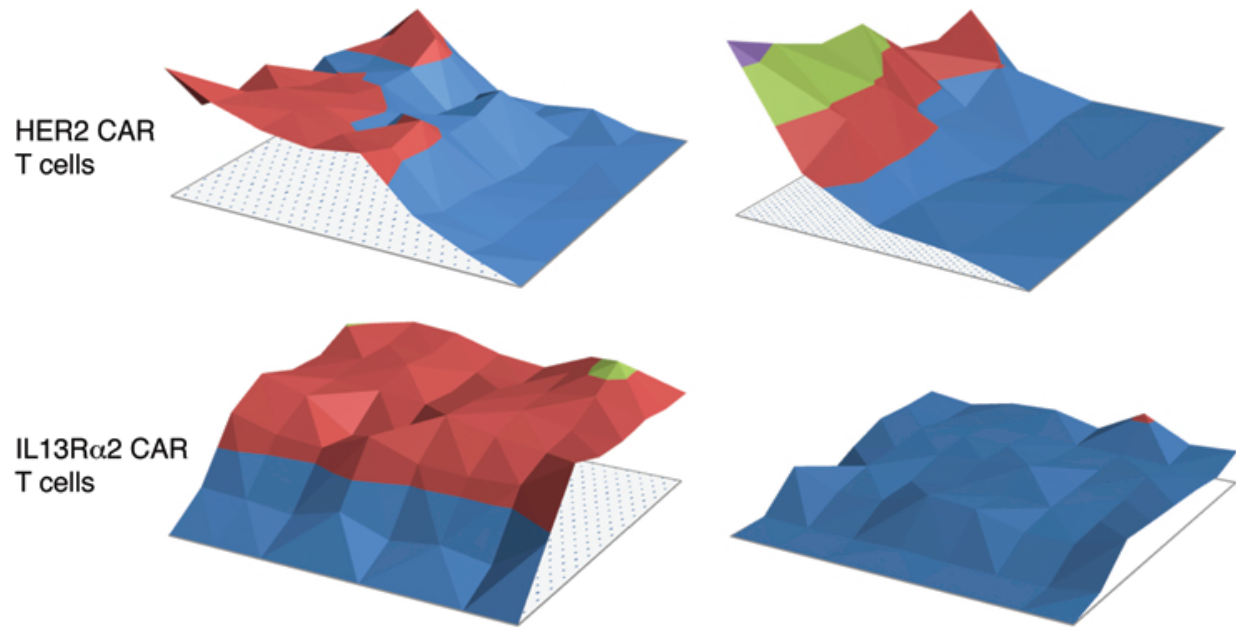

eliminate U373 glioma cells compared with other T cell effectors $(P<0.05$ at 80 hours and $P<0.005$ at 100 hours and thereafter).

We next assessed the expression of the exhaustion markers PD-1, LAG3, and TIM3 in CD4 and CD8 compartments after coculture with U373 cells for 1 week at a ratio of $1 \mathrm{~T}$ cell to 10 tumor cells. U373 glioma cells were replenished at 72 hours to ensure continuous stimulation of $\mathrm{T}$ cells. $\mathrm{T}$ cells were gated on the basis of CAR expression and compared with unstimulated CAR T cells from the same donor. $\mathrm{CD} 8^{+}$and $\mathrm{CD} 4^{+}$compartments of all CAR T cell products showed significant upregulation of surface PD-1 and LAG3 expression following coculture with U373. Both biCAR and TanCAR effectors exhibited a comparable increase in PD- 1 and LAG3 expression, similar to that seen in IL13Ra2 but not HER2 CAR T cells. TIM3 expression was significantly lower in TanCAR $\mathrm{CD} 4^{+} \mathrm{T}$ cells (Figure $\left.5 \mathrm{~B}\right)$.

TanCARs heterodimerize HER 2 and IL13R $\alpha 2$ in an enhanced bivalent immune synapse. CAR $\mathrm{T}$ cells are required to engage in an active interaction with specific target antigens on tumor cells to form an IS, which then initiates lytic killing. To investigate whether the TanCAR cells were bivalent, we determined the localization of their target antigens at the CAR T cell-GBM contact point (Figure 6A). TanCAR, IL13R $\alpha 2$ CAR, HER2 CAR, and NT T cells were incubated with U373-GBM cells, and then stained for both HER2 and IL13R $\alpha 2$. The IS was reconstituted and visualized by stacking of $1-\mu \mathrm{m}^{3}$ cubic voxels at slices spanning the $\mathrm{T}$ cell/GBM IS (Figure 6B and ref. 31). Only IL13R $\alpha 2$ or HER2 localized to the IS for the IL13R $\alpha 2$ CAR and HER2 CAR $\mathrm{T}$ cell-GBM conjugates, respectively. In contrast, both HER2 and IL13R $\alpha 2$ were found to cluster at the TanCAR IS, implying bivalency of the TanCAR T cells. Quantification of receptor accumulation at the IS revealed an increased collective clustering of both HER2 and IL13R $\alpha 2$ at the TanCAR IS compared with unispecific T cells $(P<0.005$ and $P<0.0005$, respectively; Figure $6 \mathrm{C}$ ). Further, we saw increased clustering of IL13R $\alpha 2$ at the TanCAR T cell IS, compared with the IL13R $\alpha 2$ CAR T cells $(P<$ 0.0002), and comparable HER2 clustering to that of HER2 CAR 
A

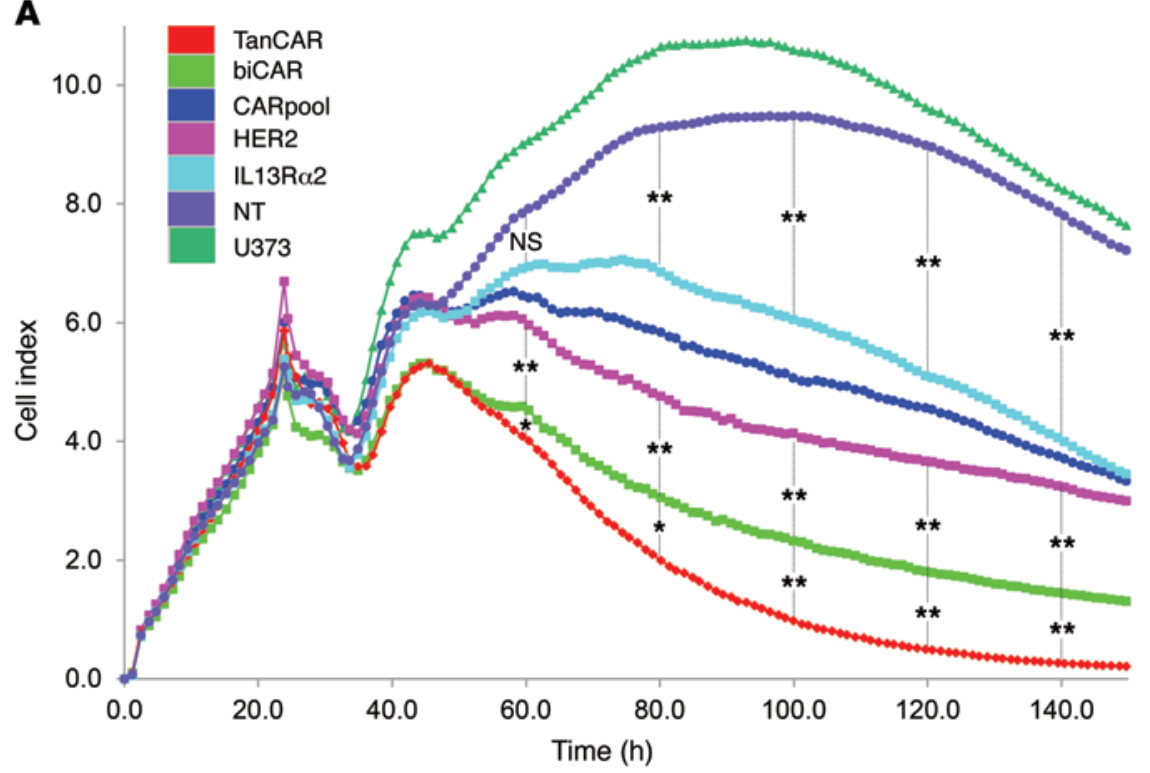

Figure 5. Assessment of sustenance of the antitumor activity of TanCAR $T$ cells and their expression of the exhaustion markers PD-1, LAG3, and TIM3. (A) Continuous graphical output of cell index values up to the 150-hour time point from U373 during incubation with TanCAR, biCAR, CARpool, HER2 CAR, and IL13R $\alpha 2$ CAR T cells and tumor only using the xCELLigence impedance system. U373 cells were seeded in electrodecoated 96-well plates (e-plates) in triplicates. T cells were added at a ratio of $1 \mathrm{~T}$ cell for each 10 U373 tumor cells 24 hours later to allow for U373 attachment and confluence. Electrical impedance was recorded continuously as an indicator of U373 density. (B) Median fluorescence intensity values for PD-1, LAC3, and TIM3 expression on the CD8 ${ }^{+}$ and $\mathrm{CD} 4+$ CAR T cell compartment before and after repeated stimulation with U373 tumor cells for 1 week. For both experiments, shown are representative data from 3 independent experiments done in triplicates. A single-step Tukey's range test was used. ${ }^{*} P<0.05,{ }^{*} P<0.005$
B

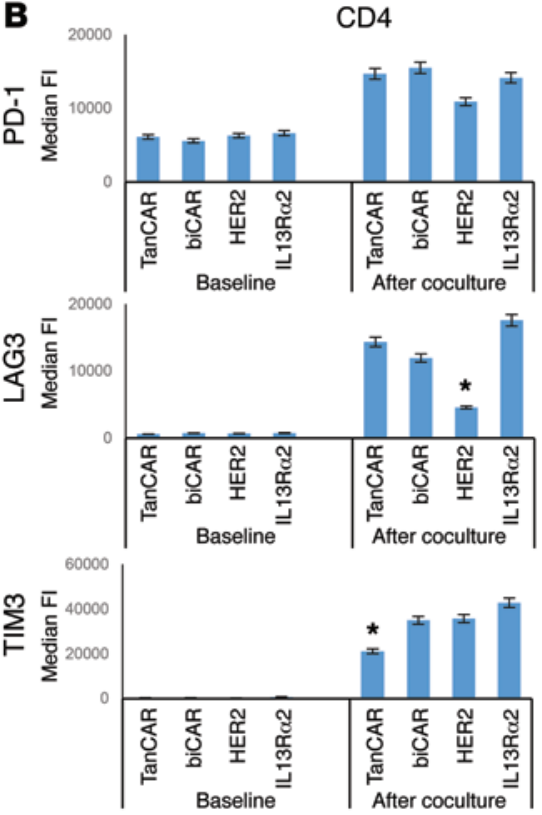

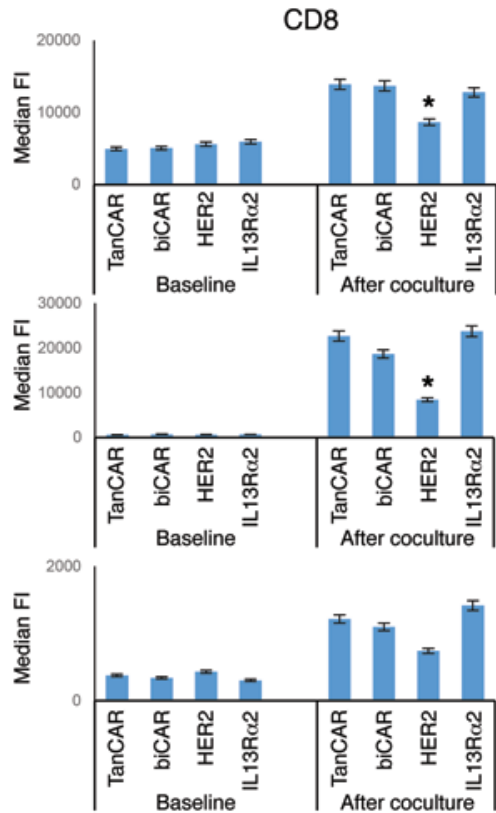

T cell IS $(P=0.8)$. NT T cells showed clustering for both IL13R $\alpha 2$ and HER2 comparable to tumor cell only $(P=0.24$ for IL13R $\alpha 2$ and $P=0.96$ for HER 2 ).

Our in silico modeling data and the ability of TanCAR molecules to mediate superadditive activation of T cells prompted us to interrogate whether TanCAR molecules could engage both HER2 and IL13R $\alpha 2$, simultaneously. Using stimulated emission depletion (STED) super-resolution microscopy that utilizes continuouswave microscopy, we imaged TanCAR T cell/U373 ISs. We were able to observe HER2 and IL13R $\alpha 2$ ligand heterodimers (Figure 7A) at the TanCAR/U373 IS at a significantly higher frequency compared with the biCAR/U373 IS (24\% vs. $<7 \%$, respectively; $P<0.001$; Figure 7B), and with a diffraction limit of less than 200 $\mathrm{nm}$. Line profiles of pixel intensity for HER2 and IL13R $\alpha 2$ showed a high enough resolution to indicate the aggregates were sufficiently proximate to support co-docking of HER2 and IL13R $\alpha 2$ to the TanCAR exodomain (Supplemental Figure 2).
To confirm and substantiate the 2-color STED findings at the molecular level, we used proximity ligation assay (PLA; Duolink) to probe for HER2-IL13R $\alpha 2$ heterodimers at the IS. PLA allows detection of protein-protein interaction within a proximity of less than $40 \mathrm{~nm}$ (Figure 7C, Supplemental Figure 3, and ref. 32). We PLA-probed TanCAR/U373 and biCAR/U373 ISs using proximity probes against HER2 (positive probe) and IL13R $\alpha 2$ (negative probe). The presence or absence of a signal (indicating proximity of the 2 ligands at $<40 \mathrm{~nm}$ ) was then detected by confocal microscopy and quantified at a per-synapse level. Mean fluorescence intensity (MFI) of the binary signals indicated a significantly increased presence of less-than-40-nm heterodimers at the TanCAR/U373 IS in comparison with the biCAR/U373 IS $(P<$ $0.0001)$. NT T cells conjugated to U373 cells were used as controls to detect background signal/noise levels (Figure 7D).

The IS mediated by the TanCAR molecule exhibited potentially advantageous qualitative and quantitative features. We 
A Bright field

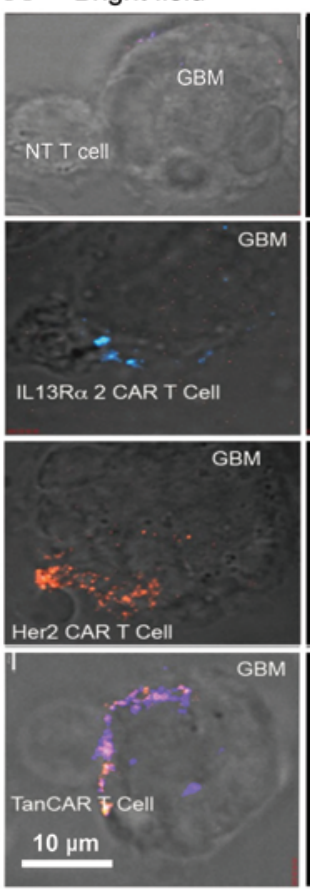

HER2
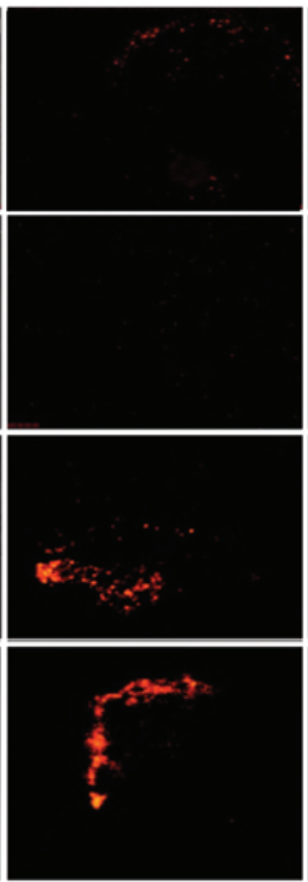

IL13R $\alpha 2$

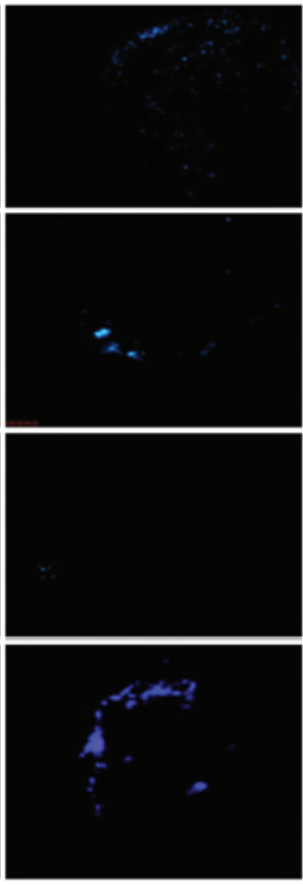

B

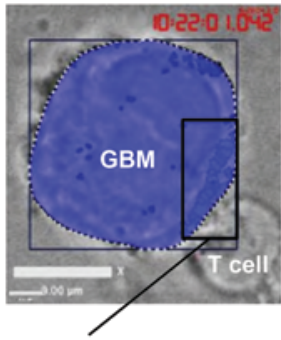

Immunological synapse (IS)

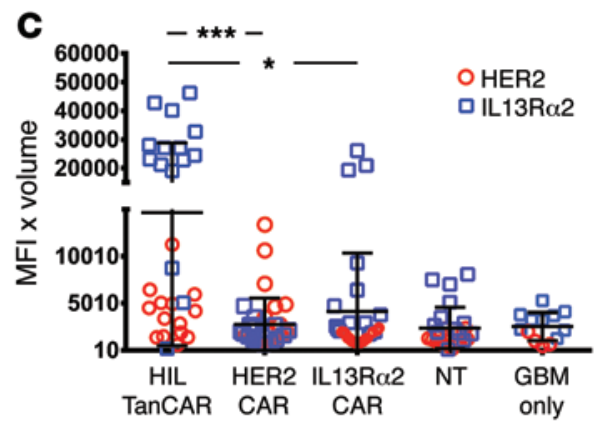

Figure 6. TanCARs recruit HER2 and IL13Ra2 to form bivalent cytolytic IS. (A) Three-dimensional confocal microscopy of fixed cells showing representative images of TanCAR T cells, IL13R $\alpha 2$ CAR T cells, HER2 CAR T cells, and NT T cells, conjugated with GBM cells. After 30 minutes at $37^{\circ} \mathrm{C}$ on Silane-coated glass slides (Thermo Fisher), conjugates were fixed and stained with anti-IL13R $\alpha 2$ (blue) and anti-HER2 (red) antibodies. (B) The IS was reconstituted in 3D, and its volume was quantified. (C) HER2 (red circles) and IL13R $\alpha 2$ (blue squares) show dual clustering at the TanCAR synapse along with significantly higher clustering of IL13R $\alpha 2$ compared with the single-CAR and NT T cells as well as compared with the normal ligand accumulation on the tumor cell surface. Cells were imaged in Z stacks on a Zeiss Axio-Observer Z1 equipped with a Yokogawa CSU10 spinning disc, Zeiss 63× 1.43 NA objective, and Hamamatsu Orca-AG camera. Images were acquired with Volocity software. Scale bar: $10 \mu \mathrm{m}$. Shown are representative data from 3 independent experiments done in triplicates. Two-tailed $t$ test and single-step Tukey's range test were performed. ${ }^{*} P<0.05,{ }^{* * *} P<0.0005$. MFI $\times$ Volume indicates the mean fluorescence intensity (MFI) of all voxels included in the $Z$ stacks of a reconstituted IS multiplied by the volume of the IS.

measured F-actin accumulation at the IS and polarization of the microtubule organizing center (MTOC), 2 well-established parameters for cellular cytolysis, to assess whether the TanCAR bivalency is associated with a functional advantage (Figure 8A). When compared with biCAR T cells, TanCAR T cells showed significantly increased MTOC polarization to the IS, indicated by reduced distance of MTOC to IS ( $n \geq 10$ synapses reconstituted, $P<0.0001)$ as well as significantly higher $\mathrm{F}$-actin accumulation at the IS ( $n \geq 20$ synapses reconstituted, $P<0.0005)$, thereby indicating a superior cytolytic potential (Figure 8B).

Collectively, our results indicate that TanCAR T cells co-recruit and co-bind HER2 and IL13R $\alpha 2$ at the IS, and that TanCAR-mediated ISs are associated with a significantly better lytic potential.

TanCAR $T$ cells improve elimination of established orthotopic GBM and mitigate antigen escape. The aforementioned data suggest TanCAR T cells may offer therapeutic superiority to unispecific CAR $\mathrm{T}$ cells and other combinatorial measures, including CARpools and biCAR T cells. We thus assessed their antitumor efficacy against established GBM xenografts in 2 scenarios of an orthotopic murine model of human GBM.

To assess the ability of TanCAR T cells to mitigate treatment failure mediated by antigen escape, we performed a "stress test" experiment wherein relatively large GBM xenografts were treated with approximately $1 \mathrm{~T}$ cell for every 30 tumor cells projected at the time of $\mathrm{T}$ cell injection. Progression-free survival (PFS), being the most stringent clinical index of treatment failure, was used as the primary outcome, and both overall survival (OS) and tumor volume using bioluminescence imaging were secondary outcomes. Tumor-only and NT T cell-treated animals served as controls. HER2 CAR T cells and IL13R $\alpha 2$ CAR T cells induced a transient but significant regression of GBM xenografts (Figure 9A and Supplemental Figure 4), with a median PFS of 14 days (range $8-19)$ and 14 days (range 7-18) after treatment, respectively $(P<$ 0.04 vs. controls; log-rank test). TanCAR T cells extended PFS to 36 days (range 32-110 days), $P=0.0007$ (Figure 9B). The median OS of HER2 CAR T cells and IL13R $\alpha 2$ CAR T cells was 53 days (range 50-56) and 55 days (range 52-57), respectively $(P<0.04$ vs. controls). TanCAR T cells extended the median OS to 86 days (range 80-100 days), $P=0.0002$ (Figure $9 \mathrm{C}$ ). NT T cells induced no appreciable tumor regression. We analyzed the expression of HER2 and IL13R $\alpha 2$ in recurrent tumors from all groups. As described above, tumors recurring after unispecific CAR treatment downregulated or eliminated target expression but maintained expression of the nontargeted antigen (20). Tumor recurrences after TanCAR $\mathrm{T}$ cell transfer were not only significantly smaller by bioluminescence imaging, they also had dim staining for both HER2 and IL13R $\alpha 2$ (Figure 9, D and E).

We have previously shown that simultaneous targeting of HER2 and IL13R $\alpha 2$ using CARpool and biCAR T cells does indeed result in improved tumor control (20). To assess the antitumor 
A
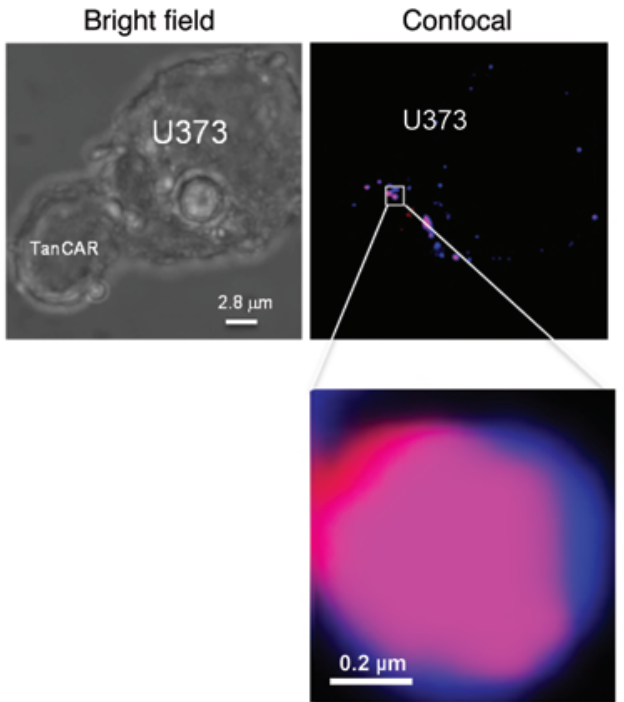

C
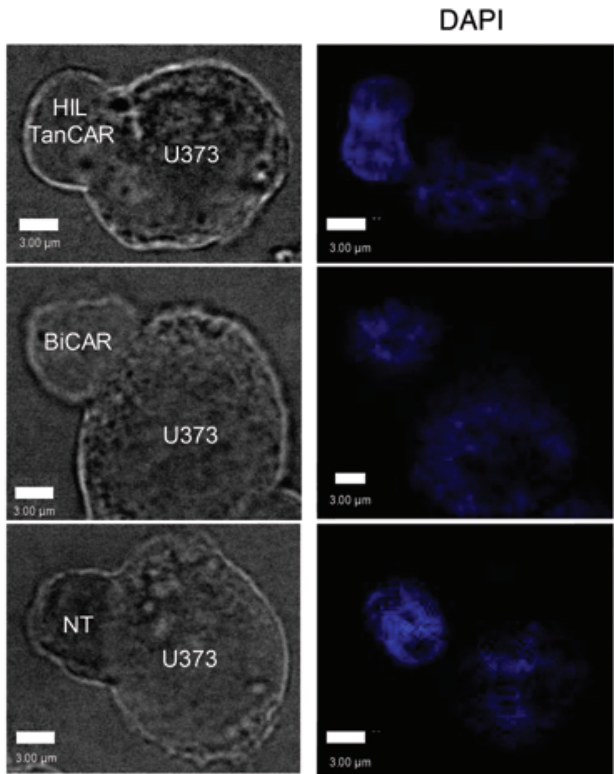

D

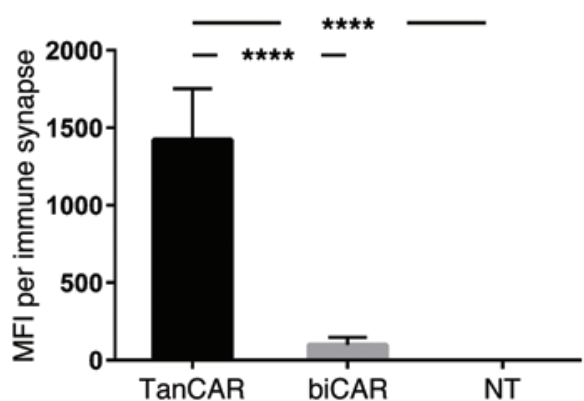

B TanCAR
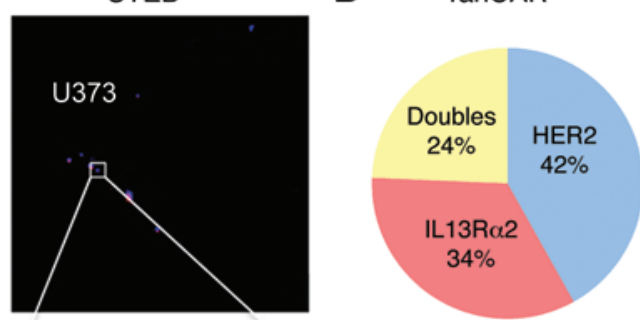

biCAR
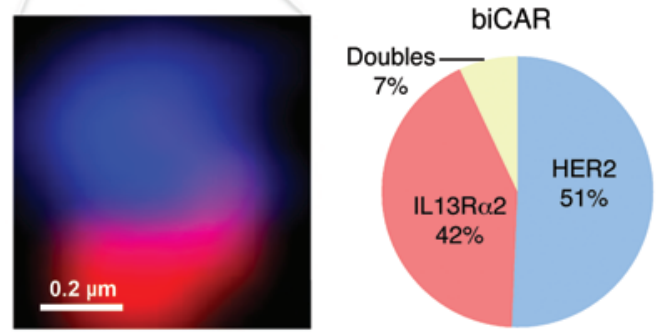

AF 561
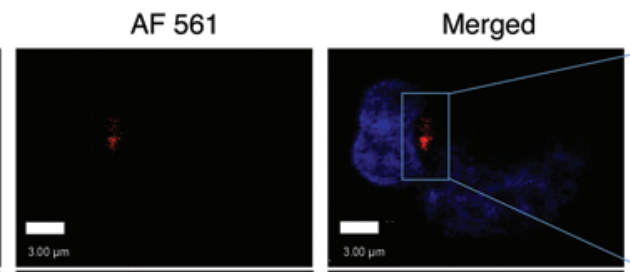

Inset
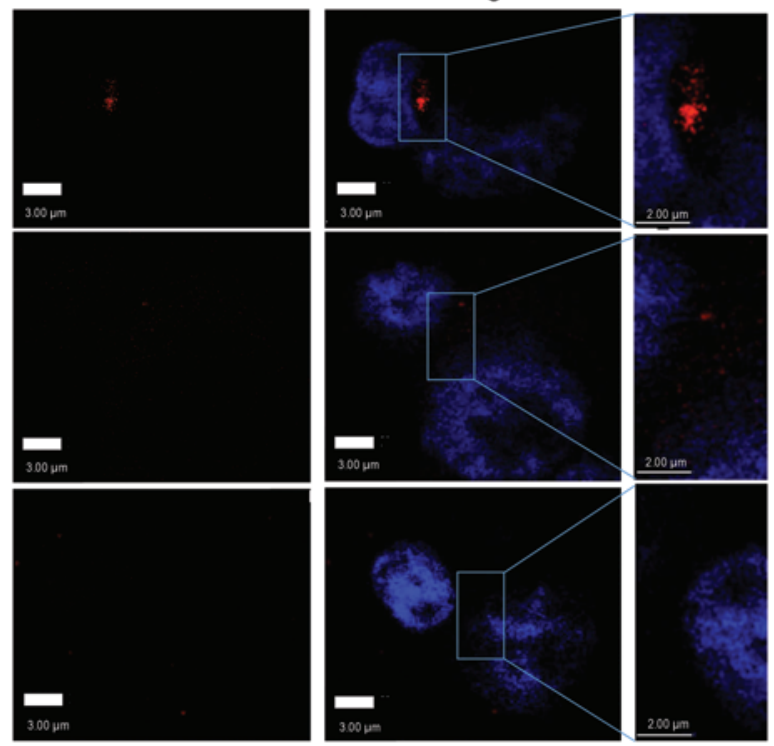

Figure 7. TanCARs heterodimerize HER2 and IL13Ra2 by engaging both target molecules simultaneously. (A) TanCAR T cells were conjugated to U373GBM cells for 10 minutes on Silane-coated glass slides and stained for HER2 (red) and IL13R 22 (blue) on the tumor surface. Conjugated CAR and U373 complexes were then imaged by dual-channel STED super-resolution microscopy. Shown is the same cell with colocalized HER2 and IL13R 22 detected by bright field (left), confocal (middle), and STED (right). A region of interest is enlarged to show greater resolution of the colocalized ligands (bottom row). (B) Pie chart distribution of single HER2 or IL13R $\alpha 2$ aggregates or dual aggregates in TanCAR and biCAR T cell conjugates to U373 cells imaged using STED microscopy is shown within a subdiffractive range of light $(<200 \mathrm{~nm})$. Full width at half maximum (FWHM) values for HER2 and IL13R $\alpha 2$ denote that the resolution using STED was $183 \mathrm{~nm}$ and $250 \mathrm{~nm}$, respectively. FWHM analysis is shown in Supplemental Figure 2. (C) Fluorescent microscopy of Duolink PLA detecting T cell-GBM interactions. U373 tumor cells were coincubated with TanCAR (top row), biCAR (middle row), and NT (bottom row) T cells. Conjugates were mounted and fixed, and the PLA was performed to detect colocalization of HER2 and IL13R $\alpha 2$ at the IS with a proximity of less than 40 nm. Images were captured with fluorescent confocal microscopy. Positive signal is indicated by red fluorescence. Scale bar: $3 \mu \mathrm{m}$. Inset scale bar: $2.0 \mu \mathrm{m}$. (D) The PLA MFI per IS was significantly higher in the TanCAR T cell-U373 interactions than in both the biCAR T cell-U373 and the NT T cell-U373 interactions, suggesting that the TanCAR is simultaneously docking with HER2 and IL13R $\alpha 2$. Shown are representative data from 4 independent experiments done in triplicates with more than 30 synapses surveyed. Two-tailed $t$ test was used. ${ }^{* * * *} P<0.00005$. 
A
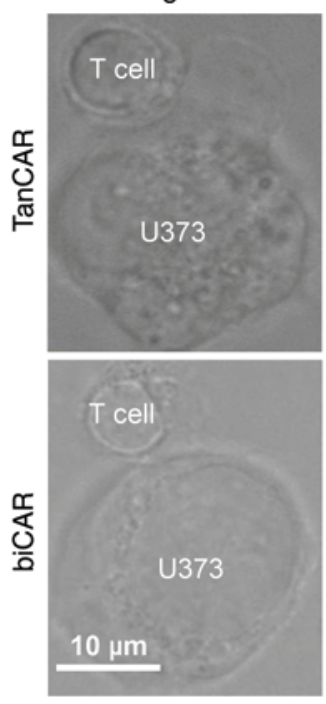

Pericentrin
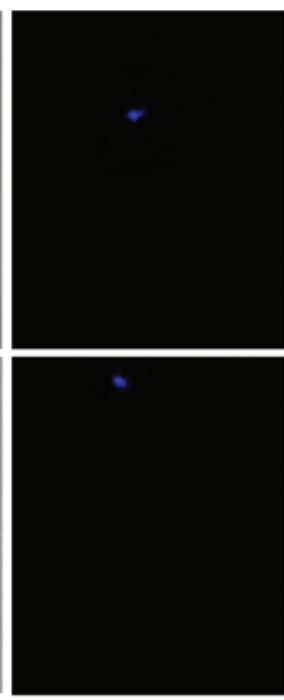

B

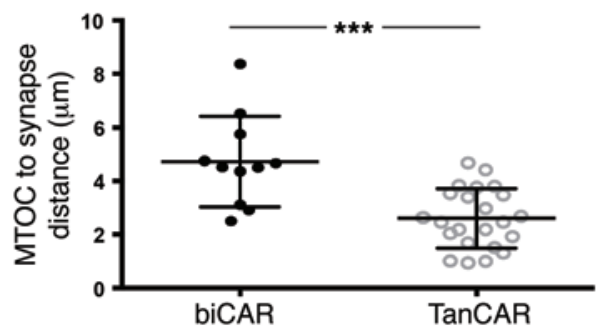

F-actin
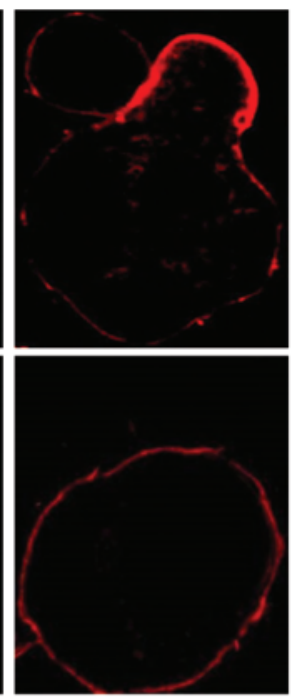

Perforin
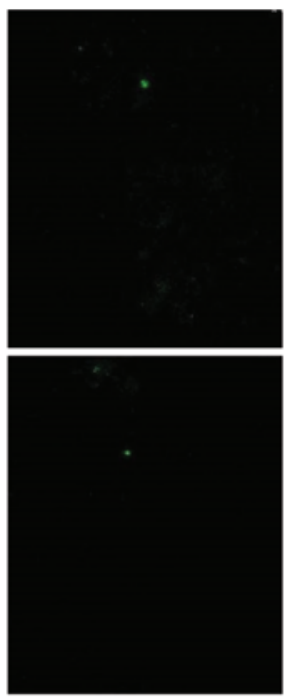

Overlay
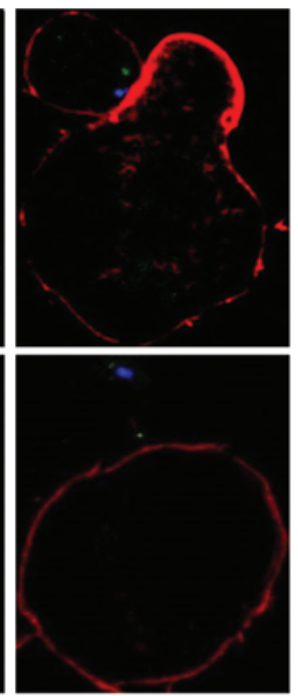

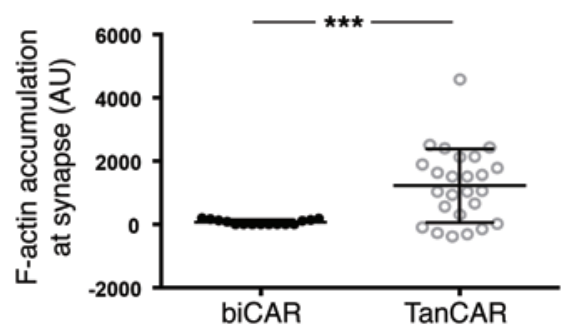

Figure 8. Increased accumulation of F-actin and increased polarization of the microtubular organizing center at the TanCAR-mediated IS. (A) Representative confocal images of TanCAR and biCAR T cells in immune conjugation with U373-GBM cells showing bright field, anti-pericentrin (blue), single-color anti-F-actin (red), anti-perforin (green), and overlay of all stains. (B) TanCAR T cells (open circles) or biCAR T cells (filled circles) were assessed for their ability to polarize to the target cell (measured by distance from the microtubular organizing center [MTOC] to the IS) and the accumulation of F-actin at the IS. Cells were imaged in Z stacks on a Leica TCS SP8 confocal microscope, Leica $\times 100$ NA objective. Images were acquired with LAS AF software (Leica) and analyzed with Volocity software. Each data point represents an individual synapse and is a collective from 2 independent experiments. ${ }^{* * *} P<0.0005$.

efficacy of TanCAR T cells in comparison with these bispecifics, we performed a second orthotopic in vivo experiment wherein established orthotopic GBM xenografts were treated with approximately $1 \mathrm{~T}$ cell for every 3 tumor cells at the time of $\mathrm{T}$ cell injection. Median OS was the primary outcome (Figure 10A). CARpool induced regression of GBM xenografts, with an OS of 52 days (range 45-66) after treatment ( $P<0.02$ vs. controls; log-rank test). biCAR T cells induced a significantly longer OS of 85 days (range 57-125 days), $P=0.001$ vs. CARpool. In contrast, the OS for the TanCAR T cells was greater than 140 days $(P<0.0001$ vs. biCAR). The study was closed at 140 days with 0 of 5 (0\%), 3 of $10(30 \%)$, and 13 of 13 (100\%) animals surviving in the CARpool, biCAR, and TanCAR groups, respectively (Figure 10B).

Collectively, these experiments demonstrate that TanCAR T cells improve control of established orthotopic GBM and mitigate antigen escape.

\section{Discussion}

We built a CAR molecule (TanCAR) specific to 2 glioma-associated antigens, HER2 and IL13R $\alpha 2$, and found that TanCAR-transduced $\mathrm{T}$ cells distinctively and effectively recognize either antigen and have superior sustained activity when both targets are encountered simultaneously. When engaged with GBM, TanCAR T cells cre- ated higher-density bispecific clustering at the IS that comprised a significant number of TanCAR-mediated HER2-IL13R $\alpha 2$ heterodimers seen on STED super-resolution microscopy and confirmed on a molecular level using PLA. This bivalent targeting of the TanCAR T cells also offset antigen escape tumor cell variants and better eliminated established tumors than unispecific CAR T cells, a pool thereof and a product of $\mathrm{T}$ cells coexpressing both CARs.

The TanCAR incorporates an FRP5-based scFv in the juxtamembrane position and a high-affinity IL-13 mutein in the distal position $(3,20)$. The expression of the exodomain in its entirety was confirmed using flow cytometric strategies specific for both domains. The distinct activation of the TanCAR $\mathrm{T}$ cells upon encounter of either target indicates that the correct folding of this complex exodomain occurred with preservation of the electrostatic properties of the individual moieties. T cell activation, measured by IFN- $\gamma$ and IL-2 secretion, was proportional to the density of target proteins immobilized to polypropylene surfaces.

The simultaneous encounter of both targets induced significantly higher cytokine secretion by TanCAR $\mathrm{T}$ cells than did exposure to either of the single targets alone. The concentration of cytokines was higher than the simple additive effect of encountering both targets individually (in contrast to an additive effect seen with biCAR T cells that declined at higher antigen concentrations). 


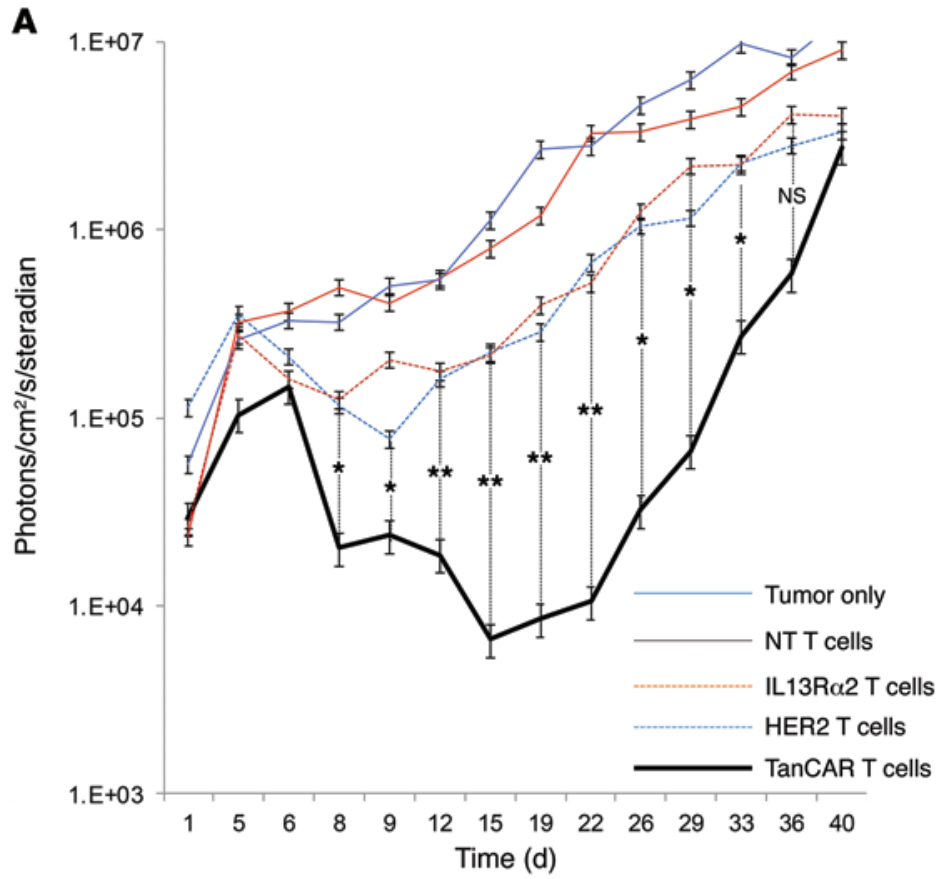

B
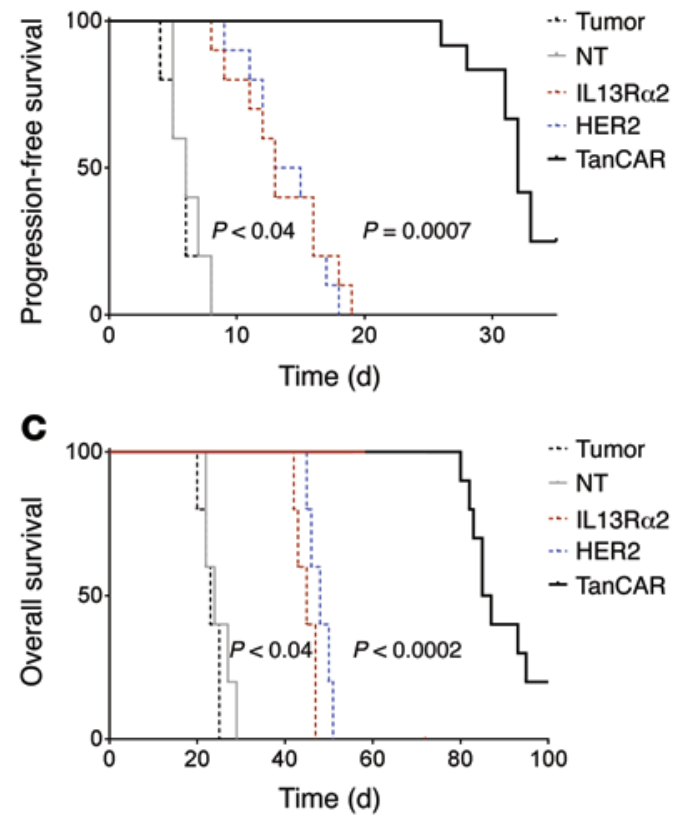

D

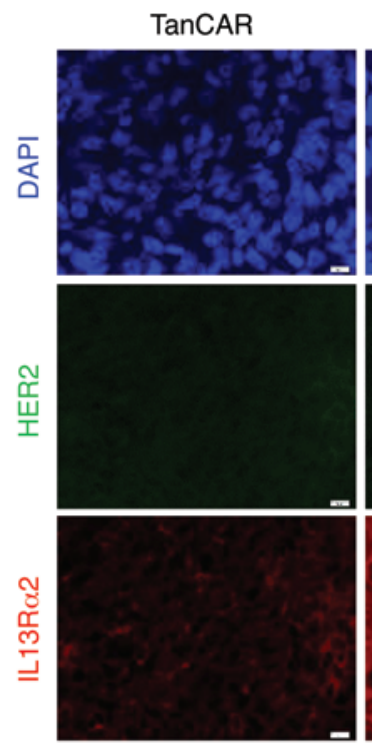

HER2 CAR

IL13R $\alpha 2$ CAR

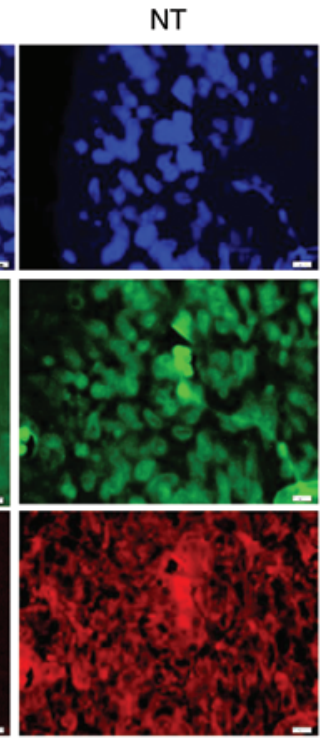

Tumor

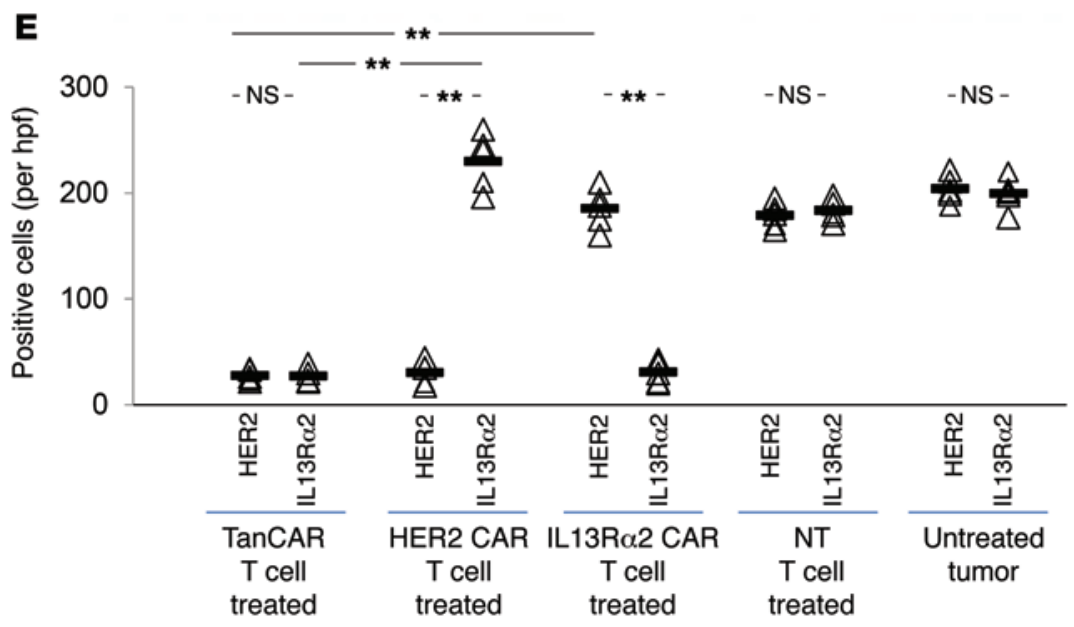


Figure 9. In vivo stress-test experiment evaluating the antitumor activity of CAR T cell products: effect on time to tumor progression, overall survival, and antigen escape. Tumors were established by stereotactic injection of $2.5 \times 10^{5}$ eGFP.Firefly luciferase-expressing U373 cells into the right frontal cortex of SCID mice. On day 8 after tumor cell injection, mice were treated with an intratumoral injection of $1 \times 10^{6}$ TanCAR T cells $(n=10)$, HER2 CAR T cells $(n=10)$, IL13R $\alpha 2$ CAR T cells $(n=10)$, or NT T cells $(n=5)$. The projected T cell/GBM cell ratio was 1:30. Untreated mice $(n=5)$ were used as controls. (A) Quantitative bioluminescence imaging done at predetermined time points to monitor tumor growth shows the group median, photons $/ \mathrm{cm}^{2} /$ second/area imaged. See Supplemental Figure 3 for representative animal images. (B) Kaplan-Meier analysis of progression-free survival closed at 40 days after the tumor was established. (C) Kaplan-Meier analysis of overall survival closed at 100 days after the tumor was established. (D) Progressive or recurrent tumors in all 5 groups were analyzed for their antigen expression pattern using coimmunofluorescence for HER2 (green) and IL13 $\alpha 2$ (red). DAPI (blue) was used for nuclear staining. Original magnification, $\times 100$. Scale bar $-20 \mu \mathrm{m}$. (E) Quantification of staining for HER2 and IL13R $\alpha 2$ of the data shown in D. Cells were counted in 5-7 hpfs. Individual values per hpf and average are shown. ${ }^{* *} P<0.005$. A single-step Tukey's range test was performed.

Moreover, while exposure to a single antigen resulted in a decline in cytokine release beyond a certain antigen density in unispecific and biCAR T cells, TanCAR T cells continued to secrete higher levels of cytokines when engaging both antigens. This superadditive cytokine production points to a reserve functional capacity of TanCAR T cells that is recruited upon simultaneous engagement.

Indeed, killing of autologous tumor targets and an enhanced cytokine release confirmed the functional superiority of TanCAR $\mathrm{T}$ cells over unispecific CAR T cells as well as biCAR T cells. A scant expression of IL13R $\alpha 2$ in UPN 3's tumor was overcome by the ability of autologous TanCAR $\mathrm{T}$ cells to recognize tumor cells expressing HER2, in this case the dominant target antigen, as well as HER2/IL13R $\alpha 2$ coexpressers. Co-docking of TanCARs to both targets - an arrangement supported by the in silico model - likely played a role in the TanCAR T cells' enhanced tumor killing. The ability to recruit target TAAs to the T cell-GBM interface was evidenced by higher-density clustering at the dual TAA IS, resulting in stronger $\mathrm{T}$ cell activation. The robust dual IS was substantiated by our in vivo experiments, in which TanCAR T cells demonstrated significantly better antitumor activity than their single-specificity CAR counterparts, as well as CARpool T cells and biCAR T cells. Tumors recurring late after TanCAR treatment lacked both targeted antigens, indicating that the improved tumor control can be explained, at least in part, by mitigating antigen escape. When we performed an "efficacy experiment" more in favor of $\mathrm{T}$ cells (albeit still at $1 \mathrm{~T}$ cell to 3 tumor cells), TanCAR T cells were able to completely and irreversibly eliminate experimental xenografts in all tumor-bearing animals.

The intrinsic enhancement of TanCAR $\mathrm{T}$ cell activity could also be attributed to their bimodal functionality. Conventional CAR T cells recognize only 1 tumor antigen. For instance, when IL13R $\alpha 2^{+}$HER $2^{+}$tumors are treated with HER2 CAR T cells, IL13R ${ }^{+}$ tumor cells survive and are positively selected, while only HER2 ${ }^{+}$ tumor cells are killed. The TanCAR molecule enables $\mathrm{T}$ cells to engage single- and double-positive populations, creating a Boolean OR argument. Perhaps an enhanced ability to engage both antigens expressed below the recognition threshold of a single CAR T cell further contributes to the broader reach of TanCAR T cells within the heterogeneous tumor subpopulations. Nevertheless, the general applicability of the TanCAR strategy to other targets and other extracellular binding domains remains to be established. Further, although unlikely, it remains unknown whether any conformational changes induced after binding of the first antigen in the tandem CAR would not change the specificity of the second $\mathrm{scFv}$ for its antigen.

Speculations about co-docking of the TanCAR molecule to both antigens on the tumor cell surface call for imaging of individual receptor-ligand interactions at the IS between effector and target cells. Imaging of clustering of proteins at the synapse, as reported here by us, has traditionally used confocal microscopy, which restricts the resolution of these images to no less than 200 $\mathrm{nm}$ approximately because of the diffraction limit of light and is therefore not enough to look at single-molecule interactions (33). Our in silico modeling data and the ability of TanCAR molecules to mediate superadditive activation of $\mathrm{T}$ cells prompted us to interrogate whether TanCAR molecules could indeed engage both HER2 and IL13R $\alpha 2$, simultaneously. While we detected colocalized aggregates of HER2 and IL13R $\alpha 2$ ligands at the TanCAR/ tumor IS, it was unclear whether these aggregates represented proximity ranges that correlated to a co-docking of the ligands to the CAR T cell. Thus, we pursued super-resolution of the IS to surpass the limit of diffraction and allow a closer look at the proximity of the individual ligands to each other at the TanCAR and biCAR ISs. STED microscopes that use continuous-wave fiber lasers can image with spatial resolution below $60 \mathrm{~nm}$ and offer the opportunity to investigate biological proximity of ligands with superior resolution. This allowed us to obtain additional insights into the docking of this new generation of CAR molecules.

The Duolink proximity ligation assay (PLA; Duolink, SigmaAldrich) has been used previously to detect protein-protein interactions, but this is its first application, to our knowledge, to detect CAR T cells binding to their target. In PLA, when 2 specific proteins are brought within a range of less than $40 \mathrm{~nm}$ of each other in the extracellular space, 2 complementary oligonucleotide sequences are able to be ligated and to initiate rolling circular DNA replication (Supplemental Figure 3). These areas of amplified DNA can be detected fluorescently and quantified. We observed significantly more clustering of these PLA signals at the immune synapse between interacting TanCAR T cells and U373 tumor cells as compared with U373 and biCAR T cells or NT $\mathrm{T}$ cells. The clustering of IL13R $\alpha 2$ and HER2 as detected by this method strongly suggests that the TanCAR T cells are co-docking with IL13R $\alpha 2$ and HER2 simultaneously on the tumor cell surface. We would expect some clustering of these tumor antigens in the interactions with biCAR $\mathrm{T}$ cells, as heterodimers that may form between IL13R $\alpha 2$ CARs and HER2 CARs could bring the 2 antigens into close enough proximity to be detected by this method. We would not expect these 2 antigens to naturally be in close proximity except by random chance on the cell surface. These hypotheses are consistent with our findings and the comparative signals detected per immune synapse.

Despite prolonged antigenic stimulation in a week-long assay, TanCAR T cells maintained their phenotype and cytolytic stamina. T cell exhaustion is a state of T cell dysfunction and sustained expression of inhibitory receptors (34). TIM3, LAG3, and 


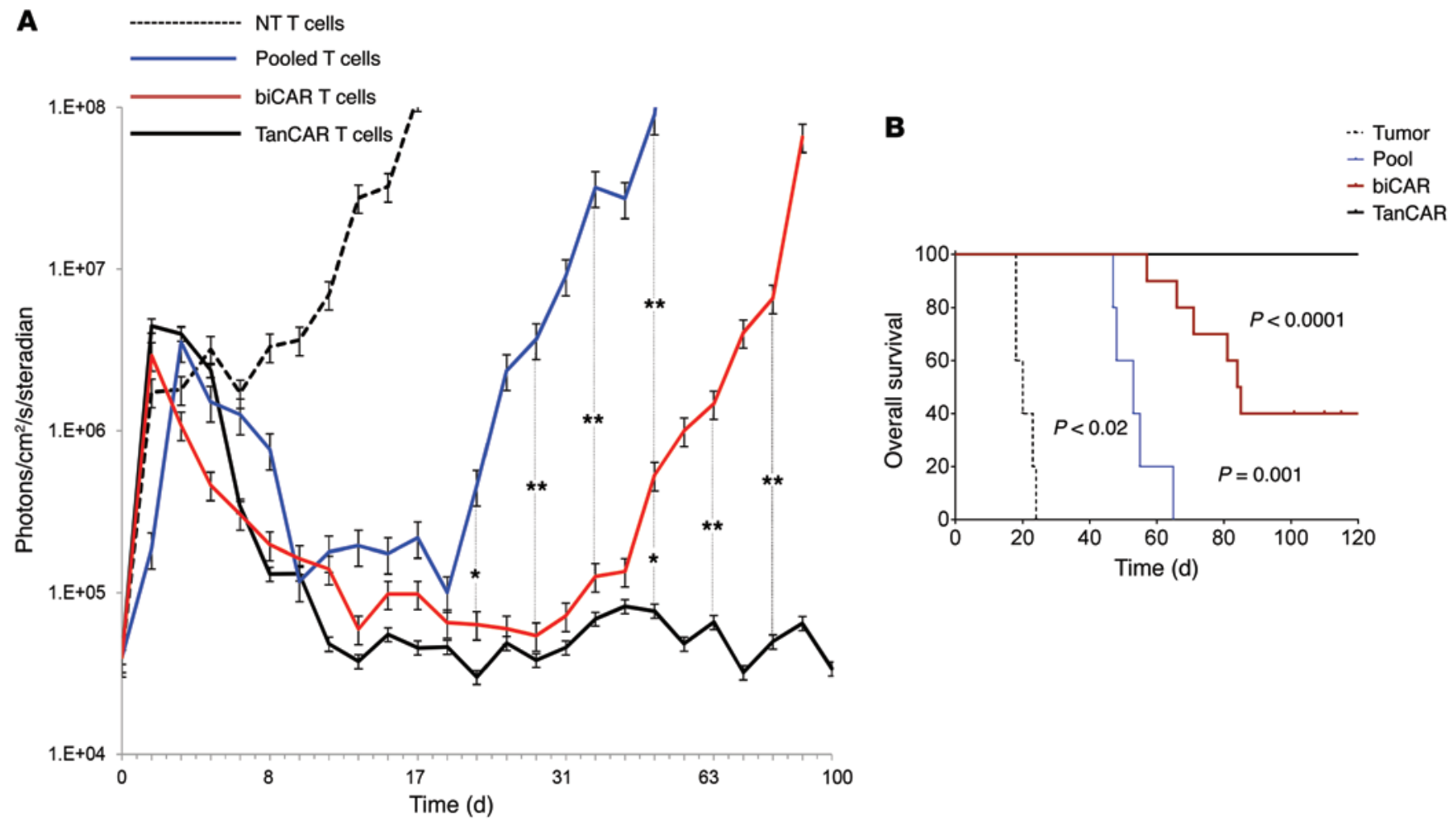

Figure 10. In vivo efficacy experiment evaluating the antitumor activity of CAR T cell products: ability to eliminate established tumors and overall survival. Tumors were established by stereotactic injection of $5 \times 10^{4}$ eGFP.Firefly luciferase-expressing U373 cells into the right frontal cortex of SCID mice. On day 5 after tumor cell injection, mice were treated with an intratumoral injection of $2 \times 10^{6}$ TanCAR T cells $(n=13)$, biCAR T cells $(n=10)$, or CARpool T cells $(n=5)$. Mice treated with NT T cells $(n=5)$ were used as controls. The projected T cell/GBM cell ratio was 1:3. (A) Quantitative bioluminescence imaging done at predetermined time points to monitor tumor growth shows the group median, photons $/ \mathrm{cm}^{2} / \mathrm{second} /$ area imaged. ${ }^{*} P<0.05$, ${ }^{* *} P<0.005$. (B) Kaplan-Meier analysis of the overall survival closed at 140 days after the tumor was established. TanCAR transduction rate was normalized to the rate of coexpression of HER2 and IL13R $\alpha 2$ in biCAR T cells and to individual CAR expression in unispecific products. $P$ values as shown in the figure were calculated using log-rank test.

PD-1 are inhibitory surface receptors that contribute to and mark a state of $\mathrm{T}$ cell exhaustion. Expression of these 3 inhibitory surface molecules was not significantly different between the biCAR and TanCAR T cells, suggesting comparable levels of activation and susceptibility to these inhibitory surface receptors. The conditional superactivation accompanied by a sustained in vitro antitumor functionality coupled with preservation of a phenotype that is not more exhausted than biCAR T cells represents a particularly interesting phenomenon in the activation dynamics of TanCAR T cells. Collectively, these could explain their enhanced functionality compared with unispecific CAR T cells and other bispecific products (CARpool and biCAR T cells) in animal experiments and perhaps point to potential superiority in the clinic.

Our work concludes that cotargeting both HER2 and IL13R $\alpha 2$ results in better tumor control, evidenced by smaller tumor volumes, increased time to tumor progression, and a better ability to eliminate established tumors (20). Two long-term follow-up animal experiments support these findings. Despite increased activation and killing, some tumors re-emerge when small $\mathrm{T}$ cell doses are used. These tumors are double-negative, indicating that TanCARs can overcome single antigen escape $(19,20)$. The delayed appearance of double-negative tumor cells, however, poses the question of what the threshold number of TAAs is that needs to be targeted and past which the tumor is not able to recover. Indeed, conceptually this would call for targeting a tumor profile rather than a single TAA. Previously, our work has in part answered this question in a mathematical model built on data sets from primary GBMs that predicts complete tumor elimination. We are currently substantiating this finding in a larger cohort of primary GBM (20). This profile may include TAAs as well as elements from the tumor microenvironment, such as the tumor stroma or tumor-associated vasculature. Nevertheless, the current work provides a clear proof of principle of the enhanced functionality and effectiveness of dual-targeting $\mathrm{T}$ cells exerting their effector function by means of a single bispecific CAR molecule.

To date, the safety of CAR T cells remains a major concern. Ontarget, off-tumor cross-reactivity with normal tissue antigens and inadvertent $\mathrm{T}$ cell stimulation and expansion remain risks. TanCAR T cells exhibit reactivity against single antigens comparable to that of conventional CAR molecules. Interestingly, they exhibit conditional superactivation when both antigens are encountered simultaneously. It is foreseeable, though, that targeting 2 antigens could increase the risk to normal tissues. To mitigate this risk, "gated" activation of CAR T cells has been described wherein an individual TAA-specific CAR molecule mediated signaling sufficient to elicit cytotoxicity and a second CAR molecule with specificity to another TAA mediated a costimulatory signal promoting full $\mathrm{T}$ cell activation (35). In cases where a TAA is shared by normal 
tissues, a CAR that provides suboptimal activation and a chimeric costimulatory receptor that recognizes a second antigen have been used to selectively "sense" and destroy tumors that express both antigens but do not affect tumors expressing either antigen alone (36-38).

Conceptually, mediating enhanced $\mathrm{T}$ cell functionality by means of a single molecule could have several advantages and pragmatic implications in addition to favorable activation dynamics (29). The introduction of a single transgene poses less risk of insertional mutagenesis, which can destabilize the $\mathrm{T}$ cell genome with the theoretical yet feared consequence of $\mathrm{T}$ cell autonomy $(39,40)$. A single genetic manipulation would be more likely to pass the stringent safety requirements of major regulatory bodies such as IRBs and the FDA and can thus be more readily furthered into the clinic. And while cellular heterogeneity is epitomized in GBM, it remains a major hurdle to the development of directed and precision therapies for most solid malignancies. Indeed, even in the broadly homogeneous B precursor malignancies, recent reports confirmed the development of CD19 escape variants after CD19 CAR T cell therapy $(14,18$, 19). Lastly, our particular choice of antigens, HER2 and IL13R $\alpha 2$, allows for a $\mathrm{T}$ cell product that is relevant not only for glioma but also for the majority of small-cell and non-small-cell lung cancers, colon cancer, and breast cancer.

In summary, targeted $\mathrm{T}$ cells are emerging as an effective and relatively nontoxic therapy for cancer. Multiple molecules, however, contribute to the overall antigenic landscape of cancers. Hence, targeting a tumor-specific profile rather than a single cancer-specific marker could result in better therapeutic efficacy. In this work, we show that a single bispecific chimeric antigen receptor molecule can effectively redirect T cells to 2 TAAs and achieve markedly enhanced tumor control in GBM.

\section{Methods}

Blood donors, primary tumor cells, and cell lines. The U373-GBM cell line was purchased from the American Type Culture Collection (ATCC). Authentication of the primary line U373 was done as previously described (2). T cells were maintained in T cell medium (250 ml RPMI-1640, 200 ml Click's medium with 10\% FCS containing 2 mmol/l GlutaMAX-I; Invitrogen). Tumor samples were processed aseptically, and primary cell cultures were initiated using DMEM with 15\% heat-inactivated FCS, 2 mM GlutaMAX-I.

Immunofluorescent staining. Formalin-fixed, paraffin-embedded sections were deparaffinized in xylene and rehydrated in ethanol. Antigen retrieval was completed by immersing of slides in $1 \times$ DAKO citrate buffer under pressure for 45 minutes. Blocking with $0.05 \%$ Tween in PBS plus 5\% donkey serum was done in humidified conditions for 1 hour at room temperature, followed by overnight incubation at $4^{\circ} \mathrm{C}$ with primary antibodies, mouse CB11 anti-HER2 in 1:25 (BioGenex), and goat anti-IL13R $\alpha 2$ in 1:500 dilution (R\&D Systems). Slides were incubated for 1 hour at room temperature in secondary antibodies diluted at 1:200 (anti-mouse Texas Red and anti-goat Cy5; Invitrogen), counterstained with nuclear stain DAPI.

Computational modeling. The structural interrogation was done using sequential modeling routines. Initial models for the IL-13 mutein and FRP5-scFv domains were generated using Phyre2. In addition, a model for the complete TanCAR exodomain was generated using the
RaptorX Web server. Docking of the IL-13 mutein model to IL13R $\alpha 2$ was refined using the Docking2 protocol from the Rosetta Online Server. Using these data, the whole TanCAR molecule was docked to individual targets and the collective data used to model a 3-way docking routine where all 3 molecules "sit" together. The final docking was selected from among the top 10 candidate dockings that had the best energy and lowest root-mean-square deviation when compared with the initial docking derived from 3LB6 (Table 1 and ref. 29). A favorable model was generated in which the orientation of the TanCAR and target molecules was maintained and the intercellular gutter was preserved.

Construction, delivery, and expression of the TanCAR-encoding transgene. The IL13R $\alpha 2$-binding IL-13 mutein was previously described (3). The HER2-specific scFv, FRP5, was previously described by Wels and colleagues (41). The modeled bispecific extracellular domain was assembled on Clone Manager (Sci-Ed Software). The designed transgene DNA sequence was optimized using the GeneOptimizer software for maximum protein production (42). The TanCAR extracellular domain was then synthesized by GeneArt Inc. using oligonucleotides, cloned into the Gateway entry vector pDONR221, and sequenceverified. This antigen recognition domain was then subcloned in frame into an SFG retroviral vector (43) containing a short hinge, the transmembrane and signaling domain of the costimulatory molecule, CD28, and the $\zeta$ signaling domain of the T cell receptor complex. The $5^{\prime}-3^{\prime}$ as well as the $3^{\prime}-5^{\prime}$ sequence of the whole construct was confirmed using single-base-pair pyrosequencing (SeqWright DNA Technology) with greater than $97 \%$ homology with the optimized construct map.

Retrovirus production and transduction of $T$ cells. To produce retroviral supernatant, human embryonic kidney (HEK) 293T cells were cotransfected with the TanCAR-encoding retroviral transfer plasmid, Peg-Pam-e plasmid encoding MoMLV gag-pol, and plasmid containing the sequence for RD114 envelope (44), using GeneJuice transfection reagent (EMD Biosciences) (44). T cells activated with anti-CD3 (OKT3) and anti-CD28 were transduced with retroviral vectors as described (2).

Flow cytometry. Analysis was done with a FACSCalibur instrument (Becton Dickinson), Kaluza software (Beckman Coulter Inc.), or FlowJo (FlowJo LLC). Cells were washed with PBS containing 2\% FBS and $0.1 \%$ sodium azide (FACS buffer; Sigma-Aldrich) before addition of the antibody. After 30-60 minutes of incubation at $4^{\circ} \mathrm{C}$ in the dark, cells were washed with FACS buffer and fixed in $0.5 \%$ paraformaldehyde for analysis.

Cell surface expression of FRP5 was detected using goat antimouse Fab fragment specific antibody conjugated with DyLight (Jackson ImmunoResearch) or a HER2.Fc chimeric protein followed by a FITC-conjugated goat anti-human Fc. IL-13 mutein was detected using an IL13R $\alpha 2$.Fc chimeric protein followed by a goat anti-human Fc fragment specific antibody conjugated with FITC (Millipore).

Analysis of cytokine production and $T$ cell expansion. T cells were cocultured with autologous GBM or U373 cells. After 24-hour incubation, conditioned culture media were collected, and levels of IFN- $\gamma$ and IL- 2 were determined by ELISA (R\&D Systems). To assess T cell activation upon encountering of immobilized target TAA, non-tissueculture-treated 24-well plates (BD Falcon) were kept overnight at $4^{\circ} \mathrm{C}$ with HER2.Fc (range 0-0.8 $\mu \mathrm{g} / \mathrm{ml}$; R\&D Systems), IL13R $\alpha 2$.Fc (range 0-10 $\mu \mathrm{g} / \mathrm{ml}$; R\&D Systems), an irrelevant target (monoclonal antiidiotype 1A7 [ref. 45]; TriGem Titan), and a nonspecific T cell receptor stimulant (OKT3). After T cell incubation for 24 hours at $37^{\circ} \mathrm{C}$, the supernatant was analyzed for IFN- $\gamma$ and IL-2. 
Cytotoxicity assays. Cytolytic activity of T cells was assessed using ${ }^{51} \mathrm{Cr}$ assays as previously described (46). The mean percentage of specific lysis of triplicate wells was calculated according to the following formula: [test release - spontaneous release] / [maximal release spontaneous release] $\times 100$.

Confocal and STED imaging of CAR synapses. For confocal microscopy, conjugates between CAR T cells and GBM cells were incubated for 45 minutes at $37^{\circ} \mathrm{C}$ and then fixed and stained for IL13R $\alpha 2$ and HER2. HER2 and IL13R $\alpha 2$ primary antibodies were subsequently detected by anti-mouse 488 and anti-goat 647 Alexa Fluor secondary antibodies (Life Technologies), respectively. Conjugates were imaged as $\mathrm{Z}$ stacks of $0.2 \mu \mathrm{m}$ thickness to cover the entire volume of the IS, determined individually for each conjugate, on a Zeiss Axio-Observer Z1 equipped with a Yokogawa CSU10 spinning disc, Zeiss $\times 631.43 \mathrm{NA}$ objective, and Hamamatsu Orca-AG camera. Images were acquired and analyzed with Volocity software (PerkinElmer). Cluster density of receptors at the synapse was calculated using the formula (volume $\times$ MFI) for an equal number of $1 \times 1 \times 1-\mu \mathrm{m}$ boxes selected to cover the IS (31). All data were graphed using GraphPad Prism software. Statistical analysis was performed using the Student's 2-tailed unpaired $t$ test. $P$ less than 0.05 was considered significant. STED microscopy was performed using a Leica TCS SP8 microscope with a $\times 100$ objective.

Proximity ligation assay (Duolink). IL13R $\alpha 2$ and HER2 antibodies were conjugated to $(+)$ and $(-)$ oligonucleotides to create PLA probes using the Duolink Probemaker MINUS and PLUS kits, respectively. For each experiment, $0.25 \times 10^{6} \mathrm{U} 373$ tumor cells plus $0.5 \times 10^{6} \mathrm{~T}$ cells were used. The slides were fixed with $4 \%$ paraformaldehyde for 10 minutes at room temperature and washed in PBS. BSA blocking solution was applied to each sample and kept at $4^{\circ} \mathrm{C}$ overnight. The PLA was performed according to the manufacturer's instructions.

Microscopy images of each slide were captured using a Zeiss Axioimager spinning disc confocal microscope at $\times 100$ magnification detecting light transmission, red fluorescence (wavelength $561 \mathrm{~nm}$ ), and DAPI fluorescence. Images were analyzed using Volocity (PerkinElmer) imaging software. Within conjugates, the area of detection of signal was delimited to the area at the IS between the respective T cell and U373 cell. The area of the immune synapse was normalized for all groups (TanCAR: mean $34.3 \mu \mathrm{m}^{2}$, range $27.4-46.79 \mu \mathrm{m}^{2}$; biCAR: mean $34.69 \mu \mathrm{m}^{2}$, range $23.57-43.61 \mu \mathrm{m}^{2}$; NT: mean $34.99 \mu \mathrm{m}^{2}$, range $26.32-45.53 \mu \mathrm{m}^{2}$ ). Detection was limited to red fluorescent signals with MFI greater than $162 \mathrm{AU}$ and area of at least $0.08 \mu \mathrm{m}^{2}$. For each condition, at least 20 conjugates were analyzed. Additionally, for each condition, background signal was analyzed by selection of single U373 tumor cells that were not interacting with any other cells. The detection area was delimited to the area near the surface of the cell similar in size to the area at the immune synapse as described above. Ten U373 cells from each slide were analyzed for this purpose. Based on the software readout of MFI and signal area, a calculation of MFI $\times$ area was made for each signal detected (AU). These were added together for each individual synapse to describe the total signal detected within 1 IS.

Impedance-based tumor cell killing assay ( $x$ CELLigence). Using the xCELLigence impedance-based system, continuous tumor cell killing was evaluated over 150 hours. U373 tumor cells were plated in a 96-well, resistor-bottomed plate at 10,000 cells per well. After 18-24 hours, 1,000 effector $\mathrm{T}$ cells (1:10 seeding ratio) were added in triplicate, at which point cell index values correlating to U373 adherence were normalized. Impedance-based measurements of the normalized cell index were collected every 15 minutes, which are determined by measurement of the impedance of current across the transistor plate caused by tumor cell adherence.

Orthotopic xenogeneic SCID mouse model of GBM. Recipient NODSCID mice were purchased from Taconic (C.B-Igh-1 ${ }^{b} /$ IcrTac-Prkd $c^{\text {scid }}$; FOX CHASE CB-17 SCID ICR). Stereotactic tumor establishment of eGFP.Firefly luciferase expressing U373 cells was performed as previously described (2). All animals had progressively growing xenografts, evidenced by progressive and exponential increments in bioluminescence signal. Each group was randomly assigned a condition of no treatment, NT T cells, HER2-specific T cells, IL13R $\alpha 2$-specific T cells, CARpool T cells, biCAR $\mathrm{T}$ cells, and TanCAR $\mathrm{T}$ cells, and received an intratumoral injection of T cells on days 5-8 (the dose and day of injection depended on the experiment as described in Results) following tumor injection. A "stress test" experiment aiming at simulating treatment failure was used to compare single-specificity $\mathrm{T}$ cells with TanCAR T cells in their ability to offset antigen escape. A separate "efficacy experiment" was used to choose which bispecific T cell product is more efficacious. Subcutaneous injection of $0.03-0.1 \mathrm{mg} / \mathrm{kg}$ buprenorphine (Buprenex RBH, England) was given for pain control.

Bioluminescence imaging. Isoflurane-anesthetized animals were imaged using the IVIS system (Xenogen Corp.) 10 minutes after i.p. injection of $150 \mathrm{mg} / \mathrm{kg}$ D-luciferin (Xenogen) as previously described (2). Animals were imaged every other day for 1 week after injections, twice per week for the next 2 weeks, and weekly thereafter until day 76. Mice were regularly examined for neurological deficits, weight loss, or signs of stress, and euthanized according to preset criteria.

Statistics. Data are presented as mean \pm SEM unless otherwise indicated in the figure legend. We used Student's $t$ test for single comparisons and Tukey's single-step test for multiple comparisons. For bioluminescence experiments, intensity signals were log-transformed and summarized using mean \pm SD at baseline and multiple subsequent time points for each mouse group. Signal intensity changes from baseline at each time point were calculated and compared using paired 2-tailed $t$ tests or Wilcoxon signed-ranks test. Log-rank test was used to compare the survival distribution between treatment groups. A $P$ value less than 0.05 was considered significant.

Study approval. All animal experiments were conducted on protocol AN3949 approved by the Baylor College of Medicine IACUC. Primary tumor cells were obtained from patients with GBM and blood samples from GBM patients and healthy donors on protocol $\mathrm{H} 15280$ approved by the IRB of Baylor College of Medicine and Houston Methodist Hospital. Written, informed consent was obtained from all patients and healthy donors.

\section{Author contributions}

All authors designed research studies, conducted experiments, acquired data, analyzed data, provided reagents, and wrote the manuscript. SN performed the PLA. MLB performed in silico modeling. MM, MM, and JO performed synapse imaging and STED.

\section{Acknowledgments}

We thank Catherine Gillespie and Sujith K. Joseph for the professional editing of the manuscript, and Nikita Aware and Ankita Shree for their technical assistance. This work was funded by Stand Up To Cancer-St. Baldrick's Pediatric Dream Team Trans- 
lational Research Grant (SU2C-AACR-DT1113). Stand Up To Cancer is a program of the Entertainment Industry Foundation administered by the American Association for Cancer Research. This work was also funded by Alex's Lemonade Stand Pediatric Cancer Foundation (ALSF) and the Alliance for Cancer Gene Therapy Inc. K. Fousek and T.T. Byrd were supported by NIH grants T32HL092332 (to Mary Estes) and T32GM088129 (to Helen Heslop) from the National Institute of General Medical Sciences. The imaging work was supported by R01AI067946 (to J.S.
Orange) and the computational biology (M. Baker) by NIH grant 8P41GM103832. This content is solely the responsibility of the authors and does not necessarily represent the official views of the National Institute of General Medical Sciences or the NIH.

Address correspondence to: Nabil Ahmed, Center for Cell and Gene Therapy, Baylor College of Medicine, 1102 Bates Street, MC 3-3320, Houston, Texas 77030, USA. Phone: 832.824.4611; E-mail:nahmed@bcm.edu.
1. Eshhar Z, Waks T, Gross G, Schindler DG. Specific activation and targeting of cytotoxic lymphocytes through chimeric single chains consisting of antibody-binding domains and the $\gamma$ or $\zeta$ subunits of the immunoglobulin and T-cell receptors. Proc Natl Acad Sci US A. 1993;90(2):720-724.

2. Ahmed N, et al. HER2-specific T cells target primary glioblastoma stem cells and induce regression of autologous experimental tumors. Clin Cancer Res. 2010;16(2):474-485.

3. Krebs S, et al. T cells redirected to interleukin$13 \mathrm{R} \alpha 2$ with interleukin-13 mutein - chimeric antigen receptors have anti-glioma activity but also recognize interleukin-13R $\alpha 1$. Cytotherapy. 2014;16(8):1121-1131.

4. Chow KK, et al. T cells redirected to EphA2 for the immunotherapy of glioblastoma. Mol Ther. 2013;21(3):629-637.

5 . Ahmed N, et al. Immunotherapy for osteosarcoma: genetic modification of $\mathrm{T}$ cells overcomes low levels of tumor antigen expression. Mol Ther. 2009;17(10):1779-1787.

6. Rainusso $\mathrm{N}$, et al. Immunotherapy targeting HER2 with genetically modified T cells eliminates tumor-initiating cells in osteosarcoma. Cancer Gene Ther. 2012;19(3):212-217.

7. Rossig C, Bollard CM, Nuchtern JG, Merchant DA, Brenner MK. Targeting of G(D2)-positive tumor cells by human $\mathrm{T}$ lymphocytes engineered to express chimeric T-cell receptor genes. Int J Cancer. 2001;94(2):228-236.

8. Ahmed N, et al. Human Epidermal Growth Factor Receptor 2 (HER2) -specific chimeric antigen receptor-modified $\mathrm{T}$ cells for the immunotherapy of HER2-positive sarcoma. J Clin Oncol. 2015;33(15):1688-1696.

9. Louis CU, et al. Antitumor activity and longterm fate of chimeric antigen receptor-positive $\mathrm{T}$ cells in patients with neuroblastoma. Blood. 2011;118(23):6050-6056.

10. Pule MA, et al. Virus-specific T cells engineered to coexpress tumor-specific receptors: persistence and antitumor activity in individuals with neuroblastoma. Nat Med. 2008;14(11):1264-1270.

11. Park JR, et al. Adoptive transfer of chimeric antigen receptor re-directed cytolytic $\mathrm{T}$ lymphocyte clones in patients with neuroblastoma. Mol Ther. 2007;15(4):825-833.

12. Brentjens RJ, et al. CD19-targeted T cells rapidly induce molecular remissions in adults with chemotherapy-refractory acute lymphoblastic leukemia. Sci Transl Med. 2013;5(177):177ra38.

13. Brentjens RJ, et al. Safety and persistence of adoptively transferred autologous CD19- targeted T-cells in patients with relapsed or chemotherapy refractory B-cell leukemias. Blood. 2011;118(18):4817-4828.

14. Grupp SA, et al. Chimeric antigen receptor-modified T-cells for acute lymphoid leukemia. $N$ EnglJ Med. 2013;368(16):1509-1518.

15. Kalos M, et al. T cells with chimeric antigen receptors have potent antitumor effects and can establish memory in patients with advanced leukemia. Sci Transl Med. 2011;3(95):95ra73.

16. Kochenderfer JN, et al. Eradication of B-lineage cells and regression of lymphoma in a patient treated with autologous T cells genetically engineered to recognize CD19. Blood. 2010;116(20):4099-4102.

17. Porter DL, Levine BL, Kalos M, Bagg A, June $\mathrm{CH}$. Chimeric antigen receptor-modified T-cells in chronic lymphoid leukemia. N Engl J Med. 2011;365(8):725-733.

18. Lee DW, et al. T cells expressing CD19 chimeric antigen receptors for acute lymphoblastic leukaemia in children and young adults: a phase 1 doseescalation trial. Lancet. 2015;385(9967):517-528.

19. Maude SL, et al. Chimeric antigen receptor $\mathrm{T}$-cells for sustained remissions in leukemia. N Engl J Med. 2014;371(16):1507-1517.

20. Hegde M, et al. Combinational targeting offsets antigen escape and enhances effector functions of adoptively transferred $\mathrm{T}$ cells in glioblastoma. Mol Ther. 2013;21(11):2087-2101.

21. Zhang JG, et al. Antigenic profiling of glioma cells to generate allogeneic vaccines or dendritic cell-based therapeutics. Clin Cancer Res. 2007;13(2 pt 1):566-575.

22. Liang Y, et al. Gene expression profiling reveals molecularly and clinically distinct subtypes of glioblastoma multiforme. Proc Natl Acad Sci US A. 2005;102(16):5814-5819.

23. Dunn GP, Old LJ, Schreiber RD. The three Es of cancer immunoediting. Annu Rev Immunol. 2004;22:329-360.

24. Nagaraj S, et al. Altered recognition of antigen is a mechanism of CD8+ T cell tolerance in cancer. Nat Med. 2007;13(7):828-835.

25. Liu G, Ying H, Zeng G, Wheeler CJ, Black KL, Yu JS. HER-2, gp100, and MAGE-1 are expressed in human glioblastoma and recognized by cytotoxic T cells. Cancer Res. 2004;64(14):4980-4986.

26. Jarboe JS, Johnson KR, Choi Y, Lonser RR, Park JK. Expression of interleukin-13 receptor $\alpha 2$ in glioblastoma multiforme: implications for targeted therapies. Cancer Res. 2007;67(17):7983-7986.

27. Wykosky J, Gibo DM, Stanton C, Debinski W. Interleukin-13 receptor $\alpha 2$, EphA2, and Fosrelated antigen 1 as molecular denominators of high-grade astrocytomas and specific targets for combinatorial therapy. Clin Cancer Res. 2008;14(1):199-208.

28. Zhang JG, et al. Tumor antigen precursor protein profiles of adult and pediatric brain tumors identify potential targets for immunotherapy. JNeurooncol. 2008;88(1):65-76.

29. Grada Z, et al. TanCAR: a novel bispecific chimeric antigen receptor for cancer immunotherapy. Mol Ther Nucleic Acids. 2013;2:e105.

30. Kahlon KS, Brown C, Cooper LJ, Raubitschek A, Forman SJ, Jensen MC. Specific recognition and killing of glioblastoma multiforme by interleukin 13-zetakine redirected cytolytic T cells. Cancer Res. 2004;64(24):9160-9166.

31. Banerjee PP, Orange JS. Quantitative measurement of F-actin accumulation at the NK cell immunological synapse. J Immunol Methods. 2010;355(1-2):1-13.

32. Söderberg O, et al. Direct observation of individual endogenous protein complexes in situ by proximity ligation. Nat Methods. 2006;3(12):995-1000.

33. Hell SW. Microscopy and its focal switch. Nat Methods. 2009;6(1):24-32.

34. Wherry EJ. T-cell exhaustion. Nat Immunol. 2011;12(6):492-499.

35. Maher J, Brentjens RJ, Gunset G, Rivière I, Sadelain M. Human T-lymphocyte cytotoxicity and proliferation directed by a single chimeric TCR $\zeta /$ CD28 receptor. Nat Biotechnol. 2002;20(1):70-75.

36. Adusumilli PS, et al. Regional delivery of mesothelin-targeted CAR T cell therapy generates potent and long-lasting CD4-dependent tumor immunity. Sci Transl Med. 2014;6(261):261ra151.

37. Duong CP, Westwood JA, Berry LJ, Darcy PK, Kershaw MH. Enhancing the specificity of T-cell cultures for adoptive immunotherapy of cancer. Immunotherapy. 2011;3(1):33-48.

38. Wilkie S, et al. Dual targeting of ErbB2 and MUC1 in breast cancer using chimeric antigen receptors engineered to provide complementary signaling. J Clin Immunol. 2012;32(5):1059-1070.

39. Hacein-Bey-Abina S, et al. Insertional oncogenesis in 4 patients after retrovirus-mediated gene therapy of SCID-X1. JClin Invest. 2008;118(9):3132-3142.

40. Craft AW, et al. A pilot study of screening for neuroblastoma in the north of England. Am J Pediatr Hematol Oncol. 1992;14(4):337-341.

41. Wels W, Harwerth IM, Zwickl M, Hardman N, Groner B, Hynes NE. Construction, bacterial expression and characterization of a bifunctional single-chain antibody-phosphatase fusion protein targeted to the human erbB-2 receptor. Biotechnology (NY). 1992;10(10):1128-1132. 
42. Raab D, Graf M, Notka F, Schödl T, Wagner R. The GeneOptimizer Algorithm: using a sliding window approach to cope with the vast sequence space in multiparameter DNA sequence optimization. Syst Synth Biol. 2010;4(3):215-225.

43. Rivière I, Brose K, Mulligan RC. Effects of retroviral vector design on expression of human adenosine deaminase in murine bone marrow transplant recipients engrafted with genetically modified cells. Proc Natl Acad Sci U S A. 1995;92(15):6733-6737.

44. Ahmed N, et al. Regression of experimental medulloblastoma following transfer of HER2-specific T cells. Cancer Res. 2007;67(12):5957-5964.
45. Yvon E, et al. Immunotherapy of metastatic melanoma using genetically engineered GD2-specific T cells. Clin Cancer Res. 2009;15(18):5852-5860. 46. Gottschalk S, et al. Generating CTLs against the subdominant Epstein-Barr virus LMP1 antigen for the adoptive immunotherapy of EBV-associated malignancies. Blood. 2003;101(5):1905-1912. 